\title{
A Convective-like Energy-Stable Open Boundary Condition for Simulations of Incompressible Flows
}

\author{
S. Dong* \\ Center for Computational \& Applied Mathematics \\ Department of Mathematics \\ Purdue University, USA
}

\begin{abstract}
We present a new energy-stable open boundary condition, and an associated numerical algorithm, for simulating incompressible flows with outflow/open boundaries. This open boundary condition ensures the energy stability of the system, even when strong vortices or backflows occur at the outflow boundary. Under certain situations it can be reduced to a form that can be analogized to the usual convective boundary condition. One prominent feature of this boundary condition is that it provides a control over the velocity on the outflow/open boundary. This is not available with the other energy-stable open boundary conditions from previous works. Our numerical algorithm treats the proposed open boundary condition based on a rotational velocity-correction type strategy. It gives rise to a Robintype condition for the discrete pressure and a Robin-type condition for the discrete velocity on the outflow/open boundary, respectively at the pressure and the velocity sub-steps. We present extensive numerical experiments on a canonical wake flow and a jet flow in open domain to test the effectiveness and performance of the method developed herein. Simulation results are compared with the experimental data as well as with other previous simulations to demonstrate the accuracy of the current method. Longtime simulations are performed for a range of Reynolds numbers, at which strong vortices and backflows occur at the outflow/open boundaries. The results show that our method is effective in overcoming the backflow instability, and that it allows for the vortices to discharge from the domain in a fairly natural fashion even at high Reynolds numbers.
\end{abstract}

Keywords: backflow instability; energy stability; outflow; open boundary condition; outflow boundary condition; velocity correction

\section{Introduction}

The current work focuses on the outflow/open boundary in incompressible flow simulations and the issue of backflow instability, which refers to the commonly-encountered numerical instability associated with strong vortices or backflows at the outflow or open boundaries. Extending our efforts on this problem [14, 12, 18], we strive to develop effective and efficient techniques to overcome the backflow instability.

A large class of flow problems involve physically-unbounded domains, such as jets, wakes, boundary layers, and other spatially-developing flows. When numerically simulating such problems, one will need to truncate the domain artificially to a finite size and impose some open (or outflow) boundary condition (OBC) on the artificial boundary. Open boundary conditions are among the most difficult and least understood issues

*Email: sdong@purdue.edu 
in incompressible flow simulations [28,63], and have commanded a sustained interest of the community for decades. Among the large volume of works accumulated so far on this problem, the traction-free condition [67, $25,19,45,3,63,30,46]$ and the convective (or radiation) boundary condition $[65,56,28,42,55,22,62,8]$ are two of the more commonly used. We refer the reader to [63] for a review of this field up to the mid-1990s, and to $[57,37,38,34,23,33,54,29,60]$ for a number of other methods developed by different researchers.

Backflow instability is a commonly encountered issue with outflows or open boundaries at moderate and high Reynolds numbers. Simulations have been observed to instantly blow up when strong vortices or backflows occur at the outflow/open boundary $[13,15,70,26]$. As pointed out in [18], a certain amount of backflow at the outflow boundary appears harmless at low Reynolds numbers, but when the Reynolds number increases beyond some moderate value, typically several hundred to a thousand depending on the geometry (e.g. between $R e=300 \sim 400$ for the flow past a square cylinder in two dimensions), this instability becomes a severe issue to numerical simulations. Commonly-used tricks for flow simulations such as increasing the grid resolution or decreasing the time step size are observed to not help with this instability [15, 18].

For certain flow problems (e.g. bluff-body wakes) one way to circumvent this difficulty is to employ a large computational domain and to place the outflow/open boundary far downstream. The idea is to allow for the vortices generated upstream to sufficiently dissipate before reaching the outflow boundary. This is feasible and computationally manageable at moderate Reynolds numbers. But this strategy does not scale with the Reynolds number $[14,18]$, because the domain size essential for numerical stability grows with increasing Reynolds number. As the Reynolds number becomes large, the needed domain size for stability can become very substantial. For example, in the three-dimensional direct numerical simulation of the flow past a circular cylinder at Reynolds number $R e=10000$ [15], a domain size with a wake region 50 times the cylinder diameter in length has been used. Such a large wake region is essential for numerical stability for that Reynolds number, even though the far wake (beyond about 10 times the cylinder diameter) is of little or no physical interest and the meshes/computations in that far region are essentially wasted.

A far more attractive approach is to devise effective open/outflow boundary conditions to overcome the backflow instability. Several such boundary conditions have been studied in the literature. By considering the weak form of the Navier-Stokes equation and symmetrization of the nonlinear term, Bruneau \& Fabrie $[6,7]$ proposed to modify the traction condition by a term $\frac{1}{2}(\mathbf{n} \cdot \mathbf{u})^{-} \mathbf{u}$, where $\mathbf{u}$ and $\mathbf{n}$ are respectively the velocity and the outward-pointing unit vector normal to the outflow boundary, and $(\mathbf{n} \cdot \mathbf{u})^{-}$is defined as $\mathbf{n} \cdot \mathbf{u}$ if $\mathbf{n} \cdot \mathbf{u}<0$ and is set to zero otherwise. We refer to e.g. [44,20] for applications of this boundary condition in later works. A traction condition containing a term $(\mathbf{n} \cdot \mathbf{u})^{-} \mathbf{u}$, which is very similar to that of $[6,7]$ but without the $\frac{1}{2}$ factor, has been employed in $[1,50,59,27,36]$. Note that a form $\beta(\mathbf{n} \cdot \mathbf{u})^{-} \mathbf{u}$ where $0<\beta<1$ has also been considered in [50]. By considering the energy balance of the system, we have proposed in [14] 
a boundary condition involving a term $\frac{1}{2}|\mathbf{u}|^{2} \mathbf{n} \Theta_{0}(\mathbf{n}, \mathbf{u})$, where $|\mathbf{u}|$ is the velocity magnitude and $\Theta_{0}(\mathbf{n}, \mathbf{u})$ is a smoothed step function about $\mathbf{n} \cdot \mathbf{u}$ (see Section 2 for definition). While the role of the term $\Theta_{0}(\mathbf{n}, \mathbf{u})$ can be compared to that of $(\mathbf{n} \cdot \mathbf{u})^{-}$discussed above, the form $\frac{1}{2}|\mathbf{u}|^{2} \mathbf{n}$ of the OBC in [14] is very different from those involving $(\mathbf{n} \cdot \mathbf{u}) \mathbf{u}$ of the previous works $[6,7,1,50,59,27,36]$. Another boundary condition developed in [4] employs a penalization of the tangential velocity derivative to allow for improved energy balance. Very recently we have proposed in [18] a family of open boundary conditions, having the characteristic that they all ensure the energy stability of the system even in situations where strong vortices of backflows occur at the outflow/open boundary. This family of boundary conditions contains those of $[6,1,27,36,14]$ as particular cases, and more importantly provides other new forms of open boundary conditions. Several of those forms have been investigated in relative detail in [18].

It is observed that, while some of the above open boundary conditions have existed in the literature for some time, their adoption in production flow simulations appears still quite limited. This is perhaps in part owing to the challenge associated with the numerical implementation of these boundary conditions. All the aforementioned boundary conditions for tackling the backflow instability couple together the velocity and the pressure, and it is not immediately clear how to implement them in numerical simulations. This seems to be exacerbated by the fact that, when these boundary conditions are originally proposed, for most of them their numerical treatments are not discussed or not adequately discussed, especially in the context of the commonly-used splitting or fractional-step type schemes for incompressible flow simulations. It is noted that in the more recent works $[14,18]$ two splitting-type schemes, respectively based on a velocity-correction type strategy [14] and a pressure-correction type strategy [18], are presented to deal with the energy-stable open boundary conditions developed therein. These algorithms de-couple the computations for the pressure and the velocity in the presence of open/outflow boundaries.

The objective of the current paper is twofold. First, we present a new energy-stable open boundary condition that is effective in overcoming the backflow instability for incompressible flow simulations. This boundary condition involves an inertia (velocity time-derivative) term, and can be shown to ensure the energy stability of the system even in the presence of backflows or vortices at the open/outflow boundary. It does not belong to the family of open boundary conditions discussed in [18]. If no backflow occurs at the outflow boundary, this boundary condition can be reduced to a form that can be analogized to the usual convective boundary condition. Hence, we refer to it as the convective-like energy-stable open boundary condition. The current open boundary condition has a prominent feature: it provides a control over the velocity at the open/outflow boundary. In contrast, the family of energy-stable open boundary conditions from [18] and the other aforementioned boundary conditions to address the backflow instability do not provide any control over the velocity at the open/outflow boundary. Therefore, as the vortices pass through the outflow/open 
boundary, the current boundary condition can lead to smoother velocity patterns in regions at or near the outflow boundary when compared to that of [18].

Second, we present an efficient numerical algorithm for treating the proposed open boundary condition. Our algorithm overall is based on a rotational velocity-correction type splitting approach, and the key issue lies in the numerical treatment of the inertia term in the open boundary condition. At the pressure sub-step our scheme leads to a Robin-type condition for the discrete pressure on the outflow boundary, and at the velocity sub-step it leads to a Robin-type condition for the discrete velocity on the outflow boundary. In contrast, the algorithms of $[14,18]$ both impose a pressure Dirichlet type condition on the outflow boundary at the pressure sub-step and a velocity Neumann type condition on the outflow boundary at the velocity sub-step. The current algorithm is simpler to implement with spectral-element (and also finite-element) type spatial discretizations, because there is no need for the projection of pressure Dirichlet data on the outflow boundary as required by the algorithms of $[14,18]$.

We would like to point out that, by using an idea analogous to that of [18], one can generalize the current open boundary condition to a family of convective-like energy-stable open boundary conditions, which will provide other new forms of OBCs; see the discussions in Section 2.1 in this regard. The numerical algorithm presented herein with no change can be applied together with this family of convective-like energy-stable OBCs.

The novelties of this work lie in two aspects: (i) the convective-like energy-stable open boundary condition, and (ii) the numerical algorithm for treating the proposed open boundary condition. The rotational velocitycorrection scheme for discretizing the Navier-Stokes equations employed here has also subtle differences than that of [14] in the numerical approximations of various terms, although both can be classified as velocitycorrection type schemes.

The open boundary condition and the numerical algorithm developed herein have been implemented and tested using high-order $C^{0}$ spectral elements for spatial discretizations [64, 41, 75]. The implementations discussed in the paper without change can also be used for finite element discretizations. It should be noted that the open boundary condition and the numerical algorithm are general and are not confined to a particular spatial discretization technique. They can also be implemented using other spatial discretization methods. 


\section{Convective-Like Energy-Stable OBC and Algorithm}

\subsection{Convective-Like Energy-Stable Open Boundary Condition}

Consider a domain $\Omega$ in two or three dimensions, and an incompressible flow contained within this domain. Let $\partial \Omega$ denote the boundary of the domain $\Omega$. This flow problem is then described by the following incompressible Navier-Stokes equations in non-dimensional form:

$$
\begin{aligned}
& \frac{\partial \mathbf{u}}{\partial t}+\mathbf{u} \cdot \nabla \mathbf{u}+\nabla p-\nu \nabla^{2} \mathbf{u}=\mathbf{f}, \\
& \nabla \cdot \mathbf{u}=0
\end{aligned}
$$

where $\mathbf{u}(\mathbf{x}, t)$ and $p(\mathbf{x}, t)$ are respectively the normalized velocity and pressure fields, $\mathbf{f}(\mathbf{x}, t)$ is an external body force, and $\mathbf{x}$ and $t$ are the spatial coordinate and time. The constant $\nu$ denotes the normalized viscosity, $\nu=\frac{1}{R e}$, where $R e$ is the Reynolds number defined by appropriately choosing a length scale and a velocity scale.

We assume that the domain boundary $\partial \Omega$ consists of two types:

$$
\partial \Omega=\partial \Omega_{d} \cup \partial \Omega_{o}, \quad \partial \Omega_{d} \cap \partial \Omega_{o}=\varnothing .
$$

In the above $\partial \Omega_{d}$ denotes the Dirichlet boundary, on which the velocity is given

$$
\mathbf{u}=\mathbf{w}(\mathbf{x}, t), \quad \text { on } \partial \Omega_{d}
$$

where $\mathbf{w}$ is the boundary velocity. On the other hand, on $\partial \Omega_{o}$ neither the velocity $\mathbf{u}$ nor the pressure $p$ is known. We refer to $\partial \Omega_{o}$ as the open (or outflow) boundary. How to deal with $\partial \Omega_{o}$ in numerical simulations is the focus of the current work.

We propose the following boundary condition for the open boundary:

$$
\nu D_{0} \frac{\partial \mathbf{u}}{\partial t}-p \mathbf{n}+\nu \mathbf{n} \cdot \nabla \mathbf{u}-\frac{1}{2}\left[|\mathbf{u}|^{2} \mathbf{n}+(\mathbf{n} \cdot \mathbf{u}) \mathbf{u}\right] \Theta_{0}(\mathbf{n}, \mathbf{u})=\mathbf{f}_{b}(\mathbf{x}, t), \quad \text { on } \partial \Omega_{o} .
$$

In the above equation, $\mathbf{n}$ is the outward-pointing unit vector normal to the boundary $\partial \Omega_{0} . D_{0}$ is a chosen

non-negative constant $\left(D_{0} \geqslant 0\right)$, which has been normalized by $\frac{1}{U_{0}}\left(U_{0}\right.$ denoting the characteristic velocity scale) and is non-dimensional. $\mathbf{f}_{b}$ is a prescribed vector function on $\partial \Omega_{o}$ for the purpose of numerical testing only, and it is set to $\mathbf{f}_{b}=0$ in actual simulations. $\Theta_{0}(\mathbf{n}, \mathbf{u})$ is a smoothed step function about $(\mathbf{n} \cdot \mathbf{u})$ given by [14],

$$
\Theta_{0}(\mathbf{n}, \mathbf{u})=\frac{1}{2}\left(1-\tanh \frac{\mathbf{n} \cdot \mathbf{u}}{\delta U_{0}}\right)
$$

where $\delta>0$ is a non-dimensional positive constant that is sufficiently small. As discussed in [14], $\delta$ controls the sharpness of the smoothed step function, and it is sharper if $\delta$ is smaller, and that the simulation result 
is not sensitive to $\delta$ when it is sufficiently small. As $\delta \rightarrow 0, \Theta_{0}(\mathbf{n}, \mathbf{u})$ approaches the step function, that is,

$$
\lim _{\delta \rightarrow 0} \Theta_{0}(\mathbf{n}, \mathbf{u})=\Theta_{s 0}(\mathbf{n}, \mathbf{u})= \begin{cases}1, & \text { if } \mathbf{n} \cdot \mathbf{u}<0 \\ 0, & \text { otherwise }\end{cases}
$$

A prominent characteristic of the open boundary condition (4) is the inertia term $\frac{\partial \mathbf{u}}{\partial t}$ involved therein. One can note that for $D_{0}=0$ the inertia term vanishes and the boundary condition (4) will be reduced to the so-called boundary condition "OBC-C" that has been studied in [18]. In the current work we concentrate on the cases with $D_{0}>0$.

The open boundary condition (4), with $\mathbf{f}_{b}=0$ and when $\delta$ is sufficiently small, ensures the energy stability of the system. To illustrate this point, we look into the energy balance equation for the system consisting of (1a) and (1b):

$$
\begin{aligned}
\frac{\partial}{\partial t} \int_{\Omega} \frac{1}{2}|\mathbf{u}|^{2}= & -\nu \int_{\Omega}\|\nabla \mathbf{u}\|^{2}+\int_{\Omega} \mathbf{f} \cdot \mathbf{u}+\int_{\partial \Omega_{d}}\left(\mathbf{n} \cdot \mathbf{T} \cdot \mathbf{u}-\frac{1}{2}|\mathbf{u}|^{2} \mathbf{n} \cdot \mathbf{u}\right) \\
& +\int_{\partial \Omega_{o}}\left(\mathbf{n} \cdot \mathbf{T} \cdot \mathbf{u}-\frac{1}{2}|\mathbf{u}|^{2} \mathbf{n} \cdot \mathbf{u}\right),
\end{aligned}
$$

where $\mathbf{T}=-p \mathbf{I}+\nu \nabla \mathbf{u}$ and $\mathbf{I}$ denotes the identity tensor. We assume that $\mathbf{f}_{b}=0$ in (4) and $\delta \rightarrow 0$ in $\Theta_{0}(\mathbf{n}, \mathbf{u})$. Then by employing the condition (4) on $\partial \Omega_{o}$, the last surface integral on the right hand side of (7) becomes

$$
\begin{aligned}
& \int_{\partial \Omega_{o}}\left(\mathbf{n} \cdot \mathbf{T} \cdot \mathbf{u}-\frac{1}{2}|\mathbf{u}|^{2} \mathbf{n} \cdot \mathbf{u}\right) \\
& =-\frac{\partial}{\partial t} \int_{\partial \Omega_{o}} \nu D_{0} \frac{1}{2}|\mathbf{u}|^{2}+\int_{\partial \Omega_{o}} \frac{1}{2}|\mathbf{u}|^{2}(\mathbf{n} \cdot \mathbf{u})\left[2 \Theta_{s 0}(\mathbf{n}, \mathbf{u})-1\right] \\
& =-\frac{\partial}{\partial t} \int_{\partial \Omega_{o}} \nu D_{0} \frac{1}{2}|\mathbf{u}|^{2}-\int_{\partial \Omega_{o}} \frac{1}{2}|\mathbf{u}|^{2}|\mathbf{n} \cdot \mathbf{u}|, \quad \text { as } \delta \rightarrow 0 .
\end{aligned}
$$

It then follows that the energy balance equation can be transformed into

$$
\begin{aligned}
& \frac{\partial}{\partial t}\left(\int_{\Omega} \frac{1}{2}|\mathbf{u}|^{2}+\nu D_{0} \int_{\partial \Omega_{o}} \frac{1}{2}|\mathbf{u}|^{2}\right) \\
& =-\nu \int_{\Omega}\|\nabla \mathbf{u}\|^{2}+\int_{\Omega} \mathbf{f} \cdot \mathbf{u}+\int_{\partial \Omega_{d}}\left(\mathbf{n} \cdot \mathbf{T} \cdot \mathbf{u}-\frac{1}{2}|\mathbf{u}|^{2} \mathbf{n} \cdot \mathbf{u}\right)-\int_{\partial \Omega_{o}} \frac{1}{2}|\mathbf{u}|^{2}|\mathbf{n} \cdot \mathbf{u}|, \quad \text { as } \delta \rightarrow 0 .
\end{aligned}
$$

Therefore, the open boundary condition given by (4), when $\mathbf{f}_{b}=0$ and $\delta$ is sufficiently small, ensures the energy stability of the system (in the absence of external forces), even if strong vortices or backflows occur (i.e. $\mathbf{n} \cdot \mathbf{u}<0$ ) on the open boundary $\partial \Omega_{o}$. Note that, because the velocity $\mathbf{u}$ is given on $\partial \Omega_{d}$, the surface integral term on $\partial \Omega_{d}$ in equation (9) will not pose a numerical instability issue.

It is instructive to compare the energy balance equations for the current open boundary condition and for the open boundary conditions introduced in [18]. Let us assume for now that there is no external body force $\mathbf{f}$ and that $\mathbf{u}=0$ on the Dirichlet boundary $\partial \Omega_{d}$. Then equation (9) implies that the sum $\left(\int_{\Omega} \frac{1}{2}|\mathbf{u}|^{2}+\nu D_{0} \int_{\partial \Omega_{o}} \frac{1}{2}|\mathbf{u}|^{2}\right)$ will not increase over time. For $D_{0}>0$, the energy balance relation provides 
an upper bound for the total energy $\int_{\Omega} \frac{1}{2}|\mathbf{u}|^{2}$ and for the quantity $\int_{\partial \Omega_{o}} \frac{1}{2}|\mathbf{u}|^{2}$ with the current open boundary condition. This provides a control over the velocity $\mathbf{u}$ on the outflow boundary $\partial \Omega_{0}$. On the other hand, with the open boundary conditions from [18], the energy balance equation involves only the total energy, and there is no control over the velocity on the outflow boundary. This is a key difference between the current open boundary condition and those from [18]. Thanks to this characteristic, the current open boundary condition can lead to qualitatively smoother velocity patterns at/near the outflow boundary as vortices pass through. This point will be illustrated in Section 3 using numerical simulations.

In addition to the open boundary condition (4), we will also consider the following boundary condition,

$$
\nu D_{0} \frac{\partial \mathbf{u}}{\partial t}-p \mathbf{n}+\nu \mathbf{n} \cdot \nabla \mathbf{u}=0, \quad \text { on } \partial \Omega_{o},
$$

or equivalently for $D_{0}>0$

$$
\frac{\partial \mathbf{u}}{\partial t}+\frac{1}{D_{0}} \frac{\partial \mathbf{u}}{\partial n}=\frac{1}{\nu D_{0}} p \mathbf{n}, \quad \text { on } \partial \Omega_{o} .
$$

The difference between this boundary condition and (4) lies in that this boundary condition does not ensure the energy stability when backflow occurs on the open boundary $\partial \Omega_{o}$. In contrast, the condition (4) ensures the energy stability even in the presence of backflows at the open boundary. Note that for $D_{0}=0$ the condition (10) will be reduced to the traction-free boundary condition. One also notes that Equation (11) is reminiscent of the usual convective boundary condition (together with $p=0$ ),

$$
\left\{\begin{array}{l}
\frac{\partial \mathbf{u}}{\partial t}+U_{c} \frac{\partial \mathbf{u}}{\partial n}=0, \quad \text { on } \partial \Omega_{o} \\
p=0, \quad \text { on } \partial \Omega_{o}
\end{array}\right.
$$

where $U_{c}$ denotes a convection velocity. Because of this resemblance to the convective boundary condition we will refer to the boundary condition (4) as a convective-like energy-stable open boundary condition.

The analogy between the current boundary condition and the usual convective boundary condition suggests that in the boundary condition (4) the parameter $\frac{1}{D_{0}}$ plays the role of a convection velocity scale at the outflow boundary. Different choices for the convection velocity in the usual convective boundary condition have been considered in a number of studies (see e.g. [63, 8]), which can provide a guide for the choice of $D_{0}$ in the boundary condition (4). For a given flow problem, one can first perform a preliminary simulation using the boundary condition (4) with $D_{0}=0$ to obtain an estimate of the convection velocity scale $U_{c}>0$ at the outflow boundary, and then carry out the actual simulation by setting $D_{0}=\frac{1}{U_{c}}$ in (4). Our numerical experiments in Section 3 indicate that the difference in the $D_{0}$ values has very little or no effect on the computed global flow quantities such as the force coefficients. The main effect of $D_{0}$ appears to be on the qualitative flow characteristics local to the outflow boundary. An improved estimate of the convection velocity (and hence an improved $D_{0}$ value in (4)) will allow the vortices or other flow features to discharge 
from the domain more smoothly and in a more natural fashion. We will look into the effects of $D_{0}$ value in the open boundary condition (4) in more detail in Section 3.

Let us briefly discuss the physical meanings of the boundary conditions (4) (assuming $\mathbf{f}_{b}=0$ and $\delta \rightarrow 0$ ), (10) and (11). These boundary conditions represent some generalized stress balance, or equivalently a Newton's law-like relation on the outflow/open boundary. As discussed in $[18,14]$, the terms $(-p \mathbf{n}+\nu \mathbf{n} \cdot \nabla \mathbf{u})$ can be considered as the fluid stress acting on $\partial \Omega_{o}$, and the terms $\frac{1}{2}|\mathbf{u}|^{2} \mathbf{n}$ and $\frac{1}{2}(\mathbf{n} \cdot \mathbf{u}) \mathbf{u}$ denote two different forms of effective stresses induced by the kinetic energy influx into the domain through $\partial \Omega_{o}$ in regions where backflow occurs. On the other hand, the term $\nu D_{0} \frac{\partial \mathbf{u}}{\partial t}$ can be considered as an effective stress associated with the fluid acceleration. In dimensional form this term can be written as $\mu \tilde{D}_{0} \frac{\partial \mathbf{u}}{\partial t}=\left(\frac{\mu \tilde{D}_{0}}{\rho}\right) \rho \frac{\partial \mathbf{u}}{\partial t}$, where $\mu$ and $\rho$ are respectively the fluid dynamic viscosity and density, $\tilde{D}_{0}$ denotes the dimensional form of $D_{0}$, and $\left(\frac{\mu \tilde{D}_{0}}{\rho}\right)$ represents a length scale. Therefore, the boundary condition (4) imposes a relation between the total of the above stresses and the fluid acceleration on the outflow boundary, which is reminiscent of the Newton's second law. On the other hand, in the boundary conditions (10) and (11) the effective stress associated with the kinetic energy influx is not considered when this relation is imposed.

Remarks By employing an idea similar to that of [18], we can come up with other forms of convectivelike energy-stable open boundary conditions. We briefly mention several of them below:

$$
\begin{aligned}
& \nu D_{0} \frac{\partial \mathbf{u}}{\partial t}-p \mathbf{n}+\nu \mathbf{n} \cdot \nabla \mathbf{u}-[(\mathbf{n} \cdot \mathbf{u}) \mathbf{u}] \Theta_{0}(\mathbf{n}, \mathbf{u})=0, \quad \text { on } \partial \Omega_{o} ; \\
& \nu D_{0} \frac{\partial \mathbf{u}}{\partial t}-p \mathbf{n}+\nu \mathbf{n} \cdot \nabla \mathbf{u}-\left[|\mathbf{u}|^{2} \mathbf{n}\right] \Theta_{0}(\mathbf{n}, \mathbf{u})=0, \quad \text { on } \partial \Omega_{o} ; \\
& \nu D_{0} \frac{\partial \mathbf{u}}{\partial t}-p \mathbf{n}+\nu \mathbf{n} \cdot \nabla \mathbf{u}-\left[\frac{1}{2}(\mathbf{n} \cdot \mathbf{u}) \mathbf{u}\right] \Theta_{0}(\mathbf{n}, \mathbf{u})=0, \quad \text { on } \partial \Omega_{o} ; \\
& \nu D_{0} \frac{\partial \mathbf{u}}{\partial t}-p \mathbf{n}+\nu \mathbf{n} \cdot \nabla \mathbf{u}-\left[\frac{1}{2}|\mathbf{u}|^{2} \mathbf{n}\right] \Theta_{0}(\mathbf{n}, \mathbf{u})=0, \quad \text { on } \partial \Omega_{o} ; \\
& \nu D_{0} \frac{\partial \mathbf{u}}{\partial t}-p \mathbf{n}+\nu \mathbf{n} \cdot \nabla \mathbf{u}-\frac{1}{4}\left[|\mathbf{u}|^{2} \mathbf{n}+(\mathbf{n} \cdot \mathbf{u}) \mathbf{u}\right] \Theta_{0}(\mathbf{n}, \mathbf{u})=0, \quad \text { on } \partial \Omega_{o} .
\end{aligned}
$$

We would also like to mention the following more general form (analogous to [18]), which contains the boundary condition (4) and those represented by (13a)-(13e) as particular cases,

$$
\nu D_{0} \frac{\partial \mathbf{u}}{\partial t}-p \mathbf{n}+\nu \mathbf{n} \cdot \nabla \mathbf{u}-\left[\left(\theta+\alpha_{2}\right) \frac{1}{2}|\mathbf{u}|^{2} \mathbf{n}+\left(1-\theta+\alpha_{1}\right) \frac{1}{2}(\mathbf{n} \cdot \mathbf{u}) \mathbf{u}\right] \Theta_{0}(\mathbf{n}, \mathbf{u})=0, \quad \text { on } \partial \Omega_{o},
$$

where $\theta, \alpha_{1}$ and $\alpha_{2}$ are constants satisfying the conditions

$$
0 \leqslant \theta \leqslant 1, \quad \alpha_{1} \geqslant 0, \quad \alpha_{2} \geqslant 0 .
$$

Note that the general form (14) ensures the energy stability of the system as $\delta \rightarrow 0$. In this case the energy 
balance relation is given by the following expression,

$$
\begin{aligned}
\frac{\partial}{\partial t} & \left(\int_{\Omega} \frac{1}{2}|\mathbf{u}|^{2}+\nu D_{0} \int_{\partial \Omega_{o}} \frac{1}{2}|\mathbf{u}|^{2}\right) \\
= & -\nu \int_{\Omega}\|\nabla \mathbf{u}\|^{2}+\int_{\Omega} \mathbf{f} \cdot \mathbf{u}+\int_{\partial \Omega_{d}}\left(\mathbf{n} \cdot \mathbf{T} \cdot \mathbf{u}-\frac{1}{2}|\mathbf{u}|^{2} \mathbf{n} \cdot \mathbf{u}\right) \\
& +\int_{\partial \Omega_{o}} \frac{1}{2}|\mathbf{u}|^{2}(\mathbf{n} \cdot \mathbf{u})\left[\left(1+\alpha_{1}+\alpha_{2}\right) \Theta_{s 0}(\mathbf{n} \cdot \mathbf{u})-1\right] \\
\leqslant & -\nu \int_{\Omega}\|\nabla \mathbf{u}\|^{2}+\int_{\Omega} \mathbf{f} \cdot \mathbf{u}+\int_{\partial \Omega_{d}}\left(\mathbf{n} \cdot \mathbf{T} \cdot \mathbf{u}-\frac{1}{2}|\mathbf{u}|^{2} \mathbf{n} \cdot \mathbf{u}\right), \quad \text { as } \delta \rightarrow 0 .
\end{aligned}
$$

Apart from the boundary conditions discussed above, we impose the following initial condition for the velocity,

$$
\mathbf{u}(\mathbf{x}, t=0)=\mathbf{u}_{i n}(\mathbf{x})
$$

where $\mathbf{u}_{i n}$ is the initial velocity field satisfying equation (1b) and compatible with the boundary condition (3) on $\partial \Omega_{d}$ at $t=0$.

\subsection{Algorithm Formulation}

The equations (1a) and (1b), the boundary condition (3) on $\partial \Omega_{d}$, and the boundary condition (4) on $\partial \Omega_{o}$, as well as the initial condition (17), together constitute the system that need to be solved in numerical simulations.

We next present an algorithm for numerically simulating this system, with emphasis on the numerical treatment of the open boundary condition (4). Let

$$
\mathbf{E}(\mathbf{n}, \mathbf{u})=\frac{1}{2}\left[|\mathbf{u}|^{2} \mathbf{n}+(\mathbf{n} \cdot \mathbf{u}) \mathbf{u}\right] \Theta_{0}(\mathbf{n}, \mathbf{u})
$$

and we re-write equation (4) into a more compact form,

$$
\nu D_{0} \frac{\partial \mathbf{u}}{\partial t}-p \mathbf{n}+\nu \mathbf{n} \cdot \nabla \mathbf{u}-\mathbf{E}(\mathbf{n}, \mathbf{u})=\mathbf{f}_{b}
$$

We will concentrate on the algorithm and implementation for $D_{0}>0$ in (19) in this and the next subsections. In section 2.4 we will briefly discuss how to deal with the case $D_{0}=0$, when the current open boundary condition is reduced to a form already studied in [18].

Let $n \geqslant 0$ denote the time step index, and $(\cdot)^{n}$ denote $(\cdot)$ at time step $n$. Define $\mathbf{u}^{0}=\mathbf{u}_{i n}$. Then, given $\mathbf{u}^{n}$ we compute $\left(p^{n+1}, \mathbf{u}^{n+1}\right)$ in a de-coupled fashion as follows:

For $p^{n+1}$ :

$$
\frac{\gamma_{0} \tilde{\mathbf{u}}^{n+1}-\hat{\mathbf{u}}}{\Delta t}+\mathbf{u}^{*, n+1} \cdot \nabla \mathbf{u}^{*, n+1}+\nabla p^{n+1}+\nu \nabla \times \nabla \times \mathbf{u}^{*, n+1}=\mathbf{f}^{n+1}
$$




$$
\begin{aligned}
& \nabla \cdot \tilde{\mathbf{u}}^{n+1}=0 \\
& \mathbf{n} \cdot \tilde{\mathbf{u}}^{n+1}=\mathbf{n} \cdot \mathbf{w}^{n+1}, \quad \text { on } \partial \Omega_{d} \\
& \nu D_{0} \frac{\gamma_{0} \tilde{\mathbf{u}}^{n+1}-\hat{\mathbf{u}}}{\Delta t} \cdot \mathbf{n}-p^{n+1}+\nu \mathbf{n} \cdot \nabla \mathbf{u}^{*, n+1} \cdot \mathbf{n}-\mathbf{n} \cdot \mathbf{E}\left(\mathbf{n}, \mathbf{u}^{*, n+1}\right)=\mathbf{f}_{b}^{n+1} \cdot \mathbf{n}, \quad \text { on } \partial \Omega_{o}
\end{aligned}
$$

For $\mathbf{u}^{n+1}$ :

$$
\begin{aligned}
& \frac{\gamma_{0} \mathbf{u}^{n+1}-\gamma_{0} \tilde{\mathbf{u}}^{n+1}}{\Delta t}-\nu \nabla^{2} \mathbf{u}^{n+1}=\nu \nabla \times \nabla \times \mathbf{u}^{*, n+1} \\
& \mathbf{u}^{n+1}=\mathbf{w}^{n+1}, \quad \text { on } \partial \Omega_{d} \\
& \nu D_{0} \frac{\gamma_{0} \mathbf{u}^{n+1}-\hat{\mathbf{u}}}{\Delta t}-p^{n+1} \mathbf{n}+\nu \mathbf{n} \cdot \nabla \mathbf{u}^{n+1}-\mathbf{E}\left(\mathbf{n}, \mathbf{u}^{*, n+1}\right)+\nu\left(\nabla \cdot \mathbf{u}^{*, n+1}\right) \mathbf{n}=\mathbf{f}_{b}^{n+1}, \quad \text { on } \partial \Omega_{o} .
\end{aligned}
$$

In the above equations, $\Delta t$ is the time step size, $\mathbf{n}$ is the outward-pointing unit vector normal to the boundary, and $\tilde{\mathbf{u}}^{n+1}$ is an auxiliary variable approximating $\mathbf{u}^{n+1}$. Let $J(J=1$ or 2$)$ denote the temporal order of accuracy of the algorithm. Then $\mathbf{u}^{*, n+1}$ is a $J$-th order explicit approximation of $\mathbf{u}^{n+1}$ given by

$$
\mathbf{u}^{*, n+1}= \begin{cases}\mathbf{u}^{n}, & J=1 \\ 2 \mathbf{u}^{n}-\mathbf{u}^{n-1}, & J=2\end{cases}
$$

The expressions $\frac{1}{\Delta t}\left(\gamma_{0} \mathbf{u}^{n+1}-\hat{\mathbf{u}}\right)$ and $\frac{1}{\Delta t}\left(\gamma_{0} \tilde{\mathbf{u}}^{n+1}-\hat{\mathbf{u}}\right)$ are approximations of $\left.\frac{\partial \mathbf{u}}{\partial t}\right|^{n+1}$ by a $J$-th order backward differentiation formula, and $\hat{\mathbf{u}}$ and $\gamma_{0}$ are given by

$$
\hat{\mathbf{u}}=\left\{\begin{array}{ll}
\mathbf{u}^{n}, & J=1, \\
2 \mathbf{u}^{n}-\frac{1}{2} \mathbf{u}^{n-1}, & J=2,
\end{array} \quad \gamma_{0}= \begin{cases}1, & J=1, \\
\frac{3}{2}, & J=2 .\end{cases}\right.
$$

Note that $\mathbf{E}(\mathbf{n}, \mathbf{u})$ is given by (18).

One can observe that the overall structure of the above algorithm represents a rotational velocitycorrection type strategy (see $[32,16,17,14])$ for de-coupling the computations of the pressure and velocity. While both belong to velocity correction-type schemes, the scheme here is somewhat different from the one of [14]. Note that in the pressure sub-step we have approximated all terms at time step $(n+1)$ with the current scheme (see equation (20a)). In contrast, in [14] the viscous and the nonlinear terms are approximated at time step $n$ rather than at $(n+1)$ in the pressure sub-step, and correspondingly some correction terms are incorporated in the subsequent velocity sub-step. The current treatment of various terms is observed to yield smaller pressure errors and comparable velocity errors compared to that of [14].

The inertia term $\nu D_{0} \frac{\partial \mathbf{u}}{\partial t}$ in the boundary condition (19) demands some care in the temporal discretization. The discrete equation $(20 \mathrm{~d})$ in the pressure sub-step stems from an inner product between the directional vector $\mathbf{n}$ and the open boundary condition (19) on the outflow boundary $\partial \Omega_{o}$. Note that the $\frac{\partial \mathbf{u}}{\partial t}$ term and the pressure term have been treated implicitly in $(20 \mathrm{~d})$, and in particular that $\frac{\partial \mathbf{u}}{\partial t}$ is approximated using $\tilde{\mathbf{u}}^{n+1}$ (instead of $\mathbf{u}^{n+1}$ ) here in this discrete equation. This point is crucial, and it effectively leads to a Robin-type condition for the pressure $p^{n+1}$ on $\partial \Omega_{o}$ because of the equation (20a). An explicit treatment of 
the $\frac{\partial \mathbf{u}}{\partial t}$ term in (20d) would seem more attractive and would result in a Dirichlet type condition for $p^{n+1}$ on $\partial \Omega_{o}$, just like in the scheme of [14]. This treatment however does not work, and is observed to be unstable unless $D_{0}$ is very small. At the velocity sub-step, in the discrete equation (21c) on $\partial \Omega_{0}$ we have treated the terms $\frac{\partial \mathbf{u}}{\partial t}$ and $\mathbf{n} \cdot \nabla \mathbf{u}$ implicitly, and note that $\frac{\partial \mathbf{u}}{\partial t}$ is approximated using $\mathbf{u}^{n+1}$ here. These numerical treatments give rise to a Robin-type condition for the discrete velocity $\mathbf{u}^{n+1}$ on $\partial \Omega_{o}$, noting that in (21c) $p^{n+1}$ is already explicitly known from the pressure sub-step. Note also that in the discrete equation (21c) an extra term $\nu(\nabla \cdot \mathbf{u}) \mathbf{n}$ has been incorporated in the formulation.

We would like to point out that the algorithmic formulation given by (20a)-(21c) can be used together with the general form of convective-like energy-stable open boundary condition (14), by setting $\mathbf{E}(\mathbf{n}, \mathbf{u})$ in the algorithm as follows

$$
\mathbf{E}(\mathbf{n}, \mathbf{u})=\left[\left(\theta+\alpha_{2}\right) \frac{1}{2}|\mathbf{u}|^{2} \mathbf{n}+\left(1-\theta+\alpha_{1}\right) \frac{1}{2}(\mathbf{n} \cdot \mathbf{u}) \mathbf{u}\right] \Theta_{0}(\mathbf{n}, \mathbf{u}) .
$$

\subsection{Implementation with $C^{0}$ Spectral Elements}

We employ $C^{0}$-continuous high-order spectral elements $[64,41,75]$ for spatial discretizations in the current work. Let us next look into how to implement the algorithm, given by (20a)-(21c), using $C^{0}$ spectral elements. The discussions in this subsection with no change also apply to $C^{0}$ finite element implementations.

As noted in several previous works $[16,17,10,14,11]$, the complication in the implementation with $C^{0}$ elements stems from the high-order derivative terms such as $\nabla \times \nabla \times \mathbf{u}$ involved in this type of algorithm, because such terms cannot be directly computed in the discrete function space of $C^{0}$ elements. We can eliminate such complications by looking into the weak forms of the algorithm. In addition, we will eliminate the auxiliary velocity $\tilde{\mathbf{u}}^{n+1}$ from the final form of the algorithm.

We first formulate the weak forms of the algorithm in the spatially continuous space. Let $q(\mathbf{x})$ denote a test function. By taking the $L^{2}$ inner product between $\nabla q$ and equation (20a) and integrating by part, we have

$$
\begin{aligned}
\int_{\Omega} \nabla p^{n+1} \cdot \nabla q+\frac{\gamma_{0}}{\Delta t} \int_{\partial \Omega_{o}} \mathbf{n} \cdot \tilde{\mathbf{u}}^{n+1} q= & \int_{\Omega} \mathbf{G}^{n+1} \cdot \nabla q-\nu \int_{\partial \Omega_{d} \cup \partial \Omega_{o}} \mathbf{n} \times \boldsymbol{\omega}^{*, n+1} \cdot \nabla q \\
& -\frac{\gamma_{0}}{\Delta t} \int_{\partial \Omega_{d}} \mathbf{n} \cdot \mathbf{w}^{n+1} q, \quad \forall q,
\end{aligned}
$$

where $\boldsymbol{\omega}=\nabla \times \mathbf{u}$ is the vorticity,

$$
\mathbf{G}^{n+1}=\mathbf{f}^{n+1}+\frac{\hat{\mathbf{u}}}{\Delta t}-\mathbf{u}^{*, n+1} \cdot \nabla \mathbf{u}^{*, n+1}
$$

and we have used equations (20b) and (20c), the divergence theorem, and the identify

$$
\int_{\Omega} \nabla \times \boldsymbol{\omega} \cdot \nabla q=\int_{\Omega} \nabla \cdot(\boldsymbol{\omega} \times \nabla q)=\int_{\partial \Omega} \mathbf{n} \times \boldsymbol{\omega} \cdot \nabla q .
$$


According to equation (20d), $\mathbf{n} \cdot \tilde{\mathbf{u}}^{n+1}$ can be expressed in terms of $p^{n+1}$ and other explicit quantities on $\partial \Omega_{o}$. We therefore can transform equation (25) into the final weak form for the pressure $p^{n+1}$,

$$
\begin{aligned}
\int_{\Omega} \nabla p^{n+1} \cdot \nabla q & +\frac{1}{\nu D_{0}} \int_{\partial \Omega_{o}} p^{n+1} q=\int_{\Omega} \mathbf{G}^{n+1} \cdot \nabla q-\nu \int_{\partial \Omega_{d} \cup \partial \Omega_{o}} \mathbf{n} \times \boldsymbol{\omega}^{*, n+1} \cdot \nabla q \\
& +\int_{\partial \Omega_{o}}\left\{-\frac{1}{\Delta t} \mathbf{n} \cdot \hat{\mathbf{u}}+\frac{1}{\nu D_{0}}\left[\nu \mathbf{n} \cdot \nabla \mathbf{u}^{*, n+1} \cdot \mathbf{n}-\mathbf{n} \cdot \mathbf{E}\left(\mathbf{n}, \mathbf{u}^{*, n+1}\right)-\mathbf{f}_{b}^{n+1} \cdot \mathbf{n}\right]\right\} q \\
& -\frac{\gamma_{0}}{\Delta t} \int_{\partial \Omega_{d}} \mathbf{n} \cdot \mathbf{w}^{n+1} q, \quad \forall q .
\end{aligned}
$$

We next sum up equations (21a) and (20a) to obtain

$$
\frac{\gamma_{0}}{\nu \Delta t} \mathbf{u}^{n+1}-\nabla^{2} \mathbf{u}^{n+1}=\frac{1}{\nu}\left(\mathbf{G}^{n+1}-\nabla p^{n+1}\right) .
$$

Let $\varphi(\mathbf{x})$ denote a test function that vanishes on $\partial \Omega_{d}$. Taking the $L^{2}$ inner product between $\varphi$ and equation (29) and integrating by part lead to

$$
\frac{\gamma_{0}}{\nu \Delta t} \int_{\Omega} \mathbf{u}^{n+1} \varphi+\int_{\Omega} \nabla \varphi \cdot \nabla \mathbf{u}^{n+1}-\int_{\partial \Omega_{0}} \mathbf{n} \cdot \nabla \mathbf{u}^{n+1} \varphi=\frac{1}{\nu} \int_{\Omega}\left(\mathbf{G}^{n+1}-\nabla p^{n+1}\right) \varphi, \quad \forall \varphi,
$$

where we have used the divergence theorem, and the fact that $\int_{\partial \Omega_{d}} \mathbf{n} \cdot \nabla \mathbf{u}^{n+1} \varphi=0$ thanks to the requirement that $\varphi=0$ on $\partial \Omega_{d}$. According to equation (21c), $\mathbf{n} \cdot \nabla \mathbf{u}^{n+1}$ can be expressed in terms of $\mathbf{u}^{n+1}$ and other explicit quantities on $\partial \Omega_{o}$. We therefore can transform (30) into the final weak form for $\mathbf{u}^{n+1}$,

$$
\begin{aligned}
\frac{\gamma_{0}}{\nu \Delta t} \int_{\Omega} \mathbf{u}^{n+1} \varphi & +\int_{\Omega} \nabla \varphi \cdot \nabla \mathbf{u}^{n+1}+\frac{\gamma_{0} D_{0}}{\Delta t} \int_{\partial \Omega_{o}} \mathbf{u}^{n+1} \varphi=\frac{1}{\nu} \int_{\Omega}\left(\mathbf{G}^{n+1}-\nabla p^{n+1}\right) \varphi \\
& +\int_{\partial \Omega_{o}}\left\{\frac{D_{0}}{\Delta t} \hat{\mathbf{u}}+\frac{1}{\nu}\left[p^{n+1} \mathbf{n}+\mathbf{E}\left(\mathbf{n}, \mathbf{u}^{*, n+1}\right)+\mathbf{f}_{b}^{n+1}-\nu\left(\nabla \cdot \mathbf{u}^{*, n+1}\right) \mathbf{n}\right]\right\} \varphi, \quad \forall \varphi .
\end{aligned}
$$

The weak forms of the algorithm in the continuum space consist of equations (28) and (31), together with the velocity Dirichlet condition (21b) on $\partial \Omega_{d}$. The auxiliary variable $\tilde{\mathbf{u}}^{n+1}$ does not appear in the weak form and is not explicitly computed. These equations in weak forms can be discretized using $C^{0}$ spectral elements (or finite elements) in a straightforward fashion.

Let $\Omega_{h}$ denote the domain $\Omega$ discretized using a spectral element mesh, and $\partial \Omega_{h}=\partial \Omega_{d h} \cup \partial \Omega_{o h}$ denote the boundary of $\Omega_{h}$, where $\partial \Omega_{d h}$ and $\partial \Omega_{o h}$ respectively represent the discretized $\partial \Omega_{d}$ and $\partial \Omega_{o}$. Let $\mathbf{X}_{h} \subset$ $\left[H^{1}\left(\Omega_{h}\right)\right]^{d}$ (where $d=2$ or 3 is the spatial dimension) denote the approximation space of the discretized velocity $\mathbf{u}_{h}^{n+1}$, and define $X_{h 0}=\left\{v \in H^{1}\left(\Omega_{h}\right):\left.v\right|_{\partial \Omega_{d h}}=0\right\}$. Let $M_{h} \subset H^{1}\left(\Omega_{h}\right)$ denote the approximation space of the discretized pressure $p^{n+1}$. We take the test function $q$ of equation (28) from $M_{h}$, and take the test function $\varphi$ of equation (31) from $X_{h 0}$. In the following let $(\cdot)_{h}$ denote the discretized version of the 
variable $(\cdot)$. Then the discretized version of equation (28) is: Find $p_{h}^{n+1} \in M_{h}$ such that

$$
\begin{aligned}
\int_{\Omega_{h}} & \nabla p_{h}^{n+1} \cdot \nabla q_{h}+\frac{1}{\nu D_{0}} \int_{\partial \Omega_{o h}} p_{h}^{n+1} q_{h}=\int_{\Omega_{h}} \mathbf{G}_{h}^{n+1} \cdot \nabla q_{h}-\nu \int_{\partial \Omega_{d h} \cup \partial \Omega_{o h}} \mathbf{n}_{h} \times \boldsymbol{\omega}_{h}^{*, n+1} \cdot \nabla q_{h} \\
& +\int_{\partial \Omega_{o h}}\left\{-\frac{1}{\Delta t} \mathbf{n}_{h} \cdot \hat{\mathbf{u}}_{h}+\frac{1}{\nu D_{0}}\left[\nu \mathbf{n}_{h} \cdot \nabla \mathbf{u}_{h}^{*, n+1} \cdot \mathbf{n}_{h}-\mathbf{n}_{h} \cdot \mathbf{E}\left(\mathbf{n}_{h}, \mathbf{u}_{h}^{*, n+1}\right)-\mathbf{f}_{b h}^{n+1} \cdot \mathbf{n}_{h}\right]\right\} q_{h} \\
& -\frac{\gamma_{0}}{\Delta t} \int_{\partial \Omega_{d h}} \mathbf{n}_{h} \cdot \mathbf{w}_{h}^{n+1} q_{h}, \quad \forall q_{h} \in M_{h} .
\end{aligned}
$$

The discretized version of equations (31) and (21b) is: Find $\mathbf{u}_{h}^{n+1} \in \mathbf{X}_{h}$ such that

$$
\begin{aligned}
\frac{\gamma_{0}}{\nu \Delta t} \int_{\Omega_{h}} \mathbf{u}_{h}^{n+1} \varphi_{h}+\int_{\Omega_{h}} \nabla \varphi_{h} \cdot \nabla \mathbf{u}_{h}^{n+1}+\frac{\gamma_{0} D_{0}}{\Delta t} \int_{\partial \Omega_{o h}} \mathbf{u}_{h}^{n+1} \varphi_{h}=\frac{1}{\nu} \int_{\Omega_{h}}\left(\mathbf{G}_{h}^{n+1}-\nabla p_{h}^{n+1}\right) \varphi_{h} \\
+\int_{\partial \Omega_{o h}}\left\{\frac{D_{0}}{\Delta t} \hat{\mathbf{u}}_{h}+\frac{1}{\nu}\left[p_{h}^{n+1} \mathbf{n}_{h}+\mathbf{E}\left(\mathbf{n}_{h}, \mathbf{u}_{h}^{*, n+1}\right)+\mathbf{f}_{b h}^{n+1}-\nu\left(\nabla \cdot \mathbf{u}_{h}^{*, n+1}\right) \mathbf{n}_{h}\right]\right\} \varphi_{h}, \\
\forall \varphi_{h} \in X_{h 0},
\end{aligned}
$$

together with

$$
\mathbf{u}_{h}^{n+1}=\mathbf{w}_{h}^{n+1}, \quad \text { on } \partial \Omega_{d h} .
$$

Our final algorithm therefore consists of the following operations within a time step: (i) Solve equation (32) for $\mathbf{p}_{h}^{n+1}$; (ii) Solve equation (33), together with the Dirichlet condition (34) on $\partial \Omega_{d h}$, for $\mathbf{u}_{h}^{n+1}$. The computations for the pressure and the velocity are de-coupled, and the computations for the three components of the velocity are also de-coupled. All the terms on the right hand sides of equations (32) and (33) can be computed directly using $C^{0}$ spectral elements. Note that the auxiliary velocity $\tilde{\mathbf{u}}^{n+1}$ is not explicitly computed.

We employ equal orders of expansion polynomials to approximate the pressure and the velocity in the current spectral-element implementation, similar to our previous works $[16,17,10,12,14,18]$. Note that in all the numerical simulations and flow tests of Section 3 we have used the same polynomial orders for the pressure and the velocity. We refer the reader to the equal-order approximations for the pressure/velocity by other researchers in the literature $[40,68,31,41,47,46,1,50]$.

We finally make some comments on a possible implementation of the algorithm (20a)-(21c) with finite difference type discretizations. One can refer to [14] for suggestions in this regard with the open boundary condition presented therein. The following discussion largely follows that of [14], but with the current open boundary condition. As suggested in [14], one can take the divergence of equation (20a), leading to a pressure Poisson-type equation

$$
\nabla^{2} p^{n+1}=\nabla \cdot\left(\mathbf{f}^{n+1}+\frac{\hat{\mathbf{u}}}{\Delta t}-\mathbf{u}^{*, n+1} \cdot \nabla \mathbf{u}^{*, n+1}\right)
$$

where equation (20b) has been used. By taking the inner product between equation (20a) and directional 
vector $\mathbf{n}$ on $\partial \Omega_{d}$ and using (20c), one attains the Neumann-type condition

$$
\frac{\partial p^{n+1}}{\partial n}=\mathbf{n} \cdot\left(\mathbf{f}^{n+1}+\frac{\hat{\mathbf{u}}}{\Delta t}-\mathbf{u}^{*, n+1} \cdot \nabla \mathbf{u}^{*, n+1}\right)-\nu \mathbf{n} \cdot \nabla \times \boldsymbol{\omega}^{*, n+1}-\frac{\gamma_{0}}{\Delta t} \mathbf{n} \cdot \mathbf{w}^{n+1}, \quad \text { on } \partial \Omega_{d} .
$$

By taking the inner product between (20a) and the directional vector $\mathbf{n}$ on $\partial \Omega_{o}$, and combining the resulting equation with (20d), one can obtain the Robin-type condition

$$
\begin{aligned}
\frac{\partial p^{n+1}}{\partial n}+\frac{1}{\nu D_{0}} p^{n+1}=\mathbf{n} \cdot\left(\mathbf{f}^{n+1}\right. & \left.-\mathbf{u}^{*, n+1} \cdot \nabla \mathbf{u}^{*, n+1}\right)-\nu \mathbf{n} \times \boldsymbol{\omega}^{*, n+1} \\
& -\frac{1}{\nu D_{0}}\left[\mathbf{f}_{b}^{n+1} \cdot \mathbf{n}+\mathbf{n} \cdot \mathbf{E}\left(\mathbf{n}, \mathbf{u}^{*, n+1}\right)-\nu \mathbf{n} \cdot \nabla \mathbf{u}^{*, n+1} \cdot \mathbf{n}\right], \quad \text { on } \partial \Omega_{o} .
\end{aligned}
$$

One can therefore solve equation (35), together with the boundary conditions (36) and (37), for the pressure $p^{n+1}$. For the velocity $\mathbf{u}^{n+1}$, one can solve equation (29), together with the Dirichlet condition (21b) and the following Robin-type condition

$$
\frac{\partial \mathbf{u}^{n+1}}{\partial n}+\frac{\gamma_{0} D_{0}}{\Delta t} \mathbf{u}^{n+1}=\frac{1}{\nu}\left[\mathbf{f}_{b}^{n+1}+\mathbf{E}\left(\mathbf{n}, \mathbf{u}^{*, n+1}\right)+p^{n+1} \mathbf{n}-\nu\left(\nabla \cdot \mathbf{u}^{*, n+1}\right) \mathbf{n}\right]+\frac{D_{0}}{\Delta t} \hat{\mathbf{u}}, \quad \text { on } \partial \Omega_{o},
$$

which stems from and is equivalent to (21c).

\subsection{The Case of $D_{0}=0$ in Open Boundary Condition}

So far we have focused on the case $D_{0}>0$ in the open boundary condition (19). In this subsection we briefly discuss the case $D_{0}=0$ in the boundary condition.

As noted in Section 2.1, with $D_{0}=0$ the boundary condition (4) is reduced to a form (so-called "OBCC") that is already studied in [18]. One can therefore employ the algorithms from [18] or [14] to treat this case. Note that the algorithm presented in [14] is with respect to the open boundary condition having a form corresponding to the so-called "OBC-E" in [18]. But the algorithm of [14] also applies to other forms of open boundary conditions given in [18].

With $D_{0}=0$ the essential difference when compared with the scheme presented in Section 2.2 lies in that, in the pressure sub-step the pressure condition on the open boundary will now become of Dirichlet type rather than Robin type, and in the velocity sub-step the velocity condition on the open boundary will become of Neumann type rather than Robin type.

We now briefly mention an algorithm for $D_{0}=0$, as an alternative to the one presented in [14]. We discretize the governing equations and the boundary conditions as follows:

For $p^{n+1}$ :

Use equations (20a), (20b), and (20c);

$$
p^{n+1}=\nu \mathbf{n} \cdot \nabla \mathbf{u}^{*, n+1} \cdot \mathbf{n}-\mathbf{n} \cdot \mathbf{E}\left(\mathbf{n}, \mathbf{u}^{*, n+1}\right)-\mathbf{f}_{b}^{n+1} \cdot \mathbf{n}, \quad \text { on } \partial \Omega_{o} .
$$


Use equations (21a) and (21b);

$$
\mathbf{n} \cdot \nabla \mathbf{u}^{n+1}=\frac{1}{\nu}\left[p^{n+1} \mathbf{n}+\mathbf{E}\left(\mathbf{n}, \mathbf{u}^{*, n+1}\right)-\nu\left(\nabla \cdot \mathbf{u}^{*, n+1}\right) \mathbf{n}+\mathbf{f}_{b}^{n+1}\right], \quad \text { on } \partial \Omega_{o} .
$$

The difference between this algorithm and that of [14] lies in that, in the pressure sub-step of this algorithm we have approximated all terms at the time step $(n+1)$ and in the velocity sub-step no correction terms are involved. On the other hand, in [14] certain terms are approximated at time step $(n+1)$ and the other terms are approximated at step $n$ in the pressure sub-step, and in the velocity sub-step several correction terms are involved as a result.

The weak forms of this algorithm can be obtained using a procedure similar to that of [14]. Let $H_{p 0}^{1}(\Omega)=$ $\left\{v \in H^{1}(\Omega):\left.v\right|_{\partial \Omega_{o}}=0\right\}$, and $q \in H_{p 0}(\Omega)$ denote the test function. Then the weak form for $p^{n+1}$ is

$$
\begin{aligned}
\int_{\Omega} \nabla p^{n+1} \cdot \nabla q= & \int_{\Omega} \mathbf{G}^{n+1} \cdot \nabla q-\nu \int_{\partial \Omega_{d} \cup \partial \Omega_{o}} \mathbf{n} \times \boldsymbol{\omega}^{*, n+1} \cdot \nabla q \\
& -\frac{\gamma_{0}}{\Delta t} \int_{\partial \Omega_{d}} \mathbf{n} \cdot \mathbf{w}^{n+1} q, \quad \forall q \in H_{p 0}^{1}(\Omega),
\end{aligned}
$$

where $\mathbf{G}^{n+1}$ is given by $(26)$. Let $H_{u 0}^{1}(\Omega)=\left\{v \in H^{1}(\Omega):\left.v\right|_{\partial \Omega_{d}}=0\right\}$, and $\varphi \in H_{u 0}^{1}(\Omega)$ denote the test function. Then the weak form for $\mathbf{u}^{n+1}$ is

$$
\begin{aligned}
\frac{\gamma_{0}}{\nu \Delta t} \int_{\Omega} \mathbf{u}^{n+1} \varphi & +\int_{\Omega} \nabla \varphi \cdot \nabla \mathbf{u}^{n+1}=\frac{1}{\nu} \int_{\Omega}\left(\mathbf{G}^{n+1}-\nabla p^{n+1}\right) \varphi \\
& +\frac{1}{\nu} \int_{\partial \Omega_{0}}\left[p^{n+1} \mathbf{n}+\mathbf{E}\left(\mathbf{n}, \mathbf{u}^{*, n+1}\right)+\mathbf{f}_{b}^{n+1}-\nu\left(\nabla \cdot \mathbf{u}^{*, n+1}\right) \mathbf{n}\right] \varphi, \quad \forall \varphi \in H_{u 0}^{1}(\Omega) .
\end{aligned}
$$

The algorithm involves the following operations within a time step: (i) Solve equation (41), together with the pressure Dirichlet condition (39a) on $\partial \Omega_{o}$, for $p^{n+1}$; (ii) Solve equation (42), together with the velocity Dirichlet condition (21b) on $\partial \Omega_{d}$, for $\mathbf{u}^{n+1}$. When imposing the pressure Dirichlet condition (39a) on $\partial \Omega_{0}$ using $C^{0}$ spectral elements (or finite elements), a projection of the pressure Dirichlet data to the $H^{1}\left(\partial \Omega_{o}\right)$ space is required because of the velocity gradient term involved in the equation; see [14] for more detailed discussions in this regard. We have implemented the above algorithm, and the numerical experiments reported in Section 3 corresponding to $D_{0}=0$ are performed using this algorithm.

\section{Representative Numerical Tests}

In this section we consider several flow problems with open/outflow boundaries and employ two-dimensional simulations to demonstrate the effectiveness and performance of the open boundary condition and the numerical algorithm developed in the previous section. At large Reynolds numbers the presence of vortices and backflows at the open/outflow boundary makes these problems very challenging to simulate. We will look 


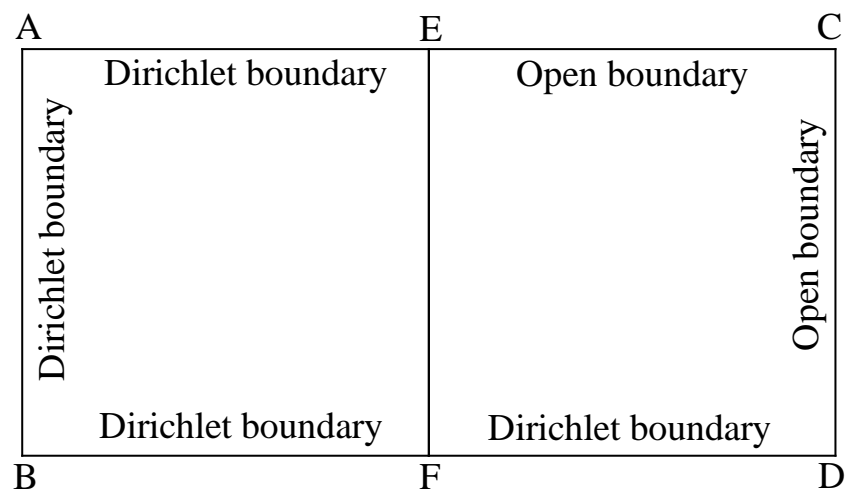

(a)

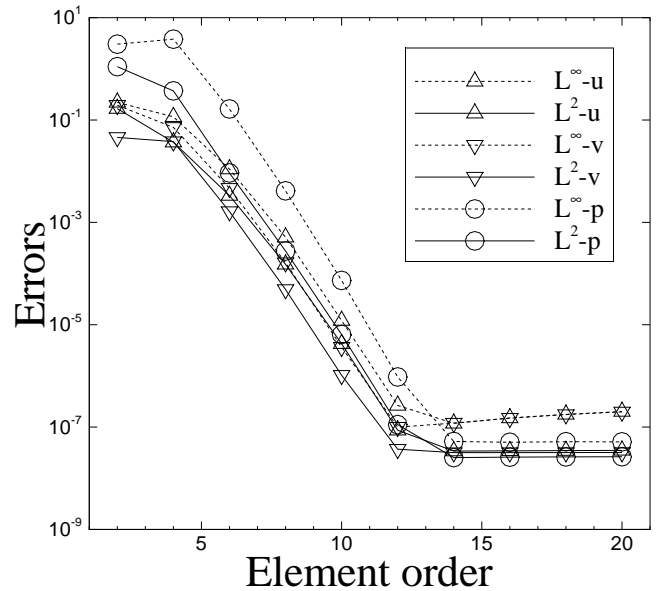

(b)

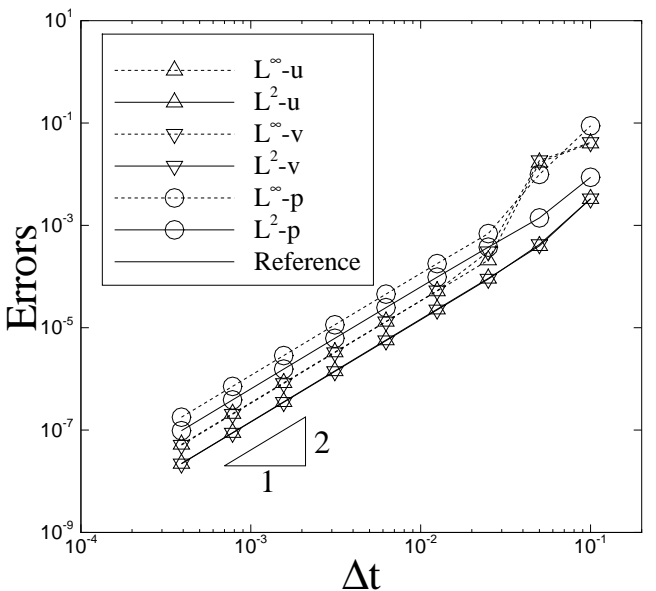

Figure 1: Spatial/temporal convergence rates: Flow configuration and boundary conditions (a), and $L^{\infty}$ and $L^{2}$ errors as a function of the element order with fixed $\Delta t=0.001$ (b) and as a function of $\Delta t$ with a fixed element order 16 (c).

into the spatial and temporal convergence rates of the algorithm, and compare current simulation results with the experimental data as well as other simulations from the literature. The results show the effectiveness of the proposed method for dealing with the backflow instability.

\subsection{Convergence Rates}

In this subsection we study the spatial and temporal convergence rates of the algorithm presented in Section 2.2 by considering an analytic solution to the Navier-Stokes equation together with the open boundary condition proposed in Section 2.1.

Figure 1(a) shows the problem setting. Consider the rectangular domain $\overline{A B D C}$ defined by $0 \leqslant x \leqslant 2$ 
and $-1 \leqslant y \leqslant 1$, and the following analytic solution to the Navier-Stokes equations, (1a) and (1b),

$$
\left\{\begin{array}{l}
u=2 \cos \pi y \sin \pi x \sin t \\
v=-2 \sin \pi y \cos \pi x \sin t \\
p=2 \sin \pi y \sin \pi x \cos t
\end{array}\right.
$$

where $\mathbf{u}=(u, v)$. We use a characteristic velocity scale $U_{0}=1$ and a non-dimensional viscosity $\nu=0.01$ for this problem. The external body force $\mathbf{f}(\mathbf{x}, t)$ in (1a) is chosen such that the expressions given by (43) satisfy the equation (1a). It is noted that the analytical solution (43) employed here has been used for the convergence tests in previous works $[14,18]$.

To simulate the problem we discretize the domain using two equal-sized quadrilateral elements $(\overline{A B F E}$ and $\overline{E F D C}$ ) along the $x$ direction. On the sides $\overline{B D}, \overline{A B}$ and $\overline{A E}$ we impose the Dirichlet condition (3), where the boundary velocity $\mathbf{w}(\mathbf{x}, t)$ is chosen according to the analytic expressions given in (43). On the sides $\overline{E C}$ and $\overline{C D}$ we impose the open boundary condition (4), in which we set $D_{0}=1.0$ and $\delta=\frac{1}{20}$ and $\mathbf{f}_{b}(\mathbf{x}, t)$ is chosen such that the velocity and pressure expressions given by (43) satisfy the condition (4) at these boundaries.

We integrate the Navier-Stokes equations (1a)-(1b) using the scheme presented in 2.2 in time from $t=0$ to $t=t_{f}$ ( $t_{f}$ to be specified below). Then we compute the errors of the numerical solution at $t=t_{f}$ against the analytic expression given in (43). The element order or the time step size $\Delta t$ has been varied systematically, and the errors are collected and monitored to study the convergence behavior of the method.

Let us first look into the spatial convergence behavior. In this group of tests we fix the time step size at $\Delta t=0.001$ and the integration time at $t_{f}=0.1$ (100 time steps), and then vary the element order systematically between 2 and 20. The numerical errors corresponding to each element order have been computed and monitored. Figure 1(b) shows the $L^{\infty}$ and $L^{2}$ errors of the velocity and the pressure as a function of the element order from these tests. As the element order increases but within order 12, all the numerical errors are observed to decrease exponentially. When the element order increases to 12 and beyond, the error curves are observed to level off at a level $\sim 10^{-7}$ for this problem. The saturation of the total numerical error is because the temporal truncation error becomes dominant when the element order becomes large. These results demonstrate the spatial exponential convergence rate of our method.

The temporal convergence behavior of the method is demonstrated by Figure 1(c), in which we plot the $L^{\infty}$ and $L^{2}$ errors of the flow variables as a function of the time step size $\Delta t$. In this group of tests the integration time is fixed at $t_{f}=0.5$, the element order is fixed at 16 , and $\Delta t$ is varied systematically between $\Delta t=0.1$ and $\Delta t=0.000390625$. The convergence appears somewhat not very regular when $\Delta t$ is above 0.025 , especially in terms of the $L^{\infty}$ error norms. As $\Delta t$ decreases below 0.025 , one can observe a second-order convergence rate in time for all the flow variables. 


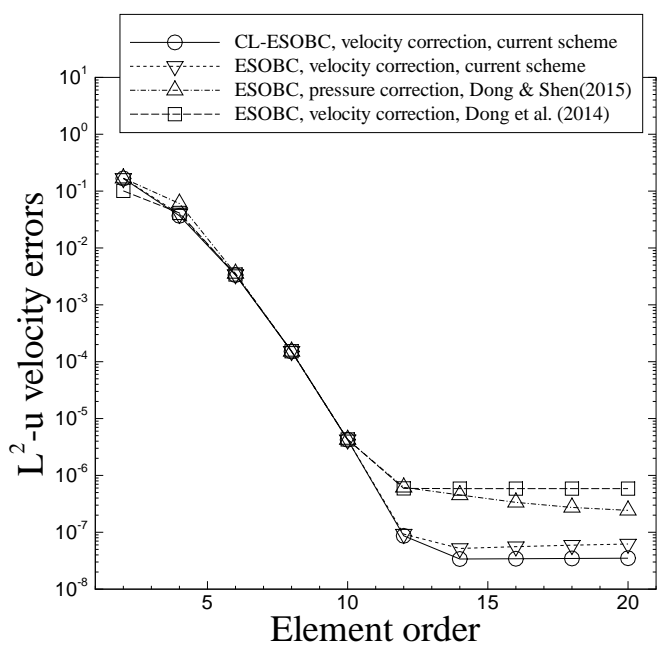

Element order

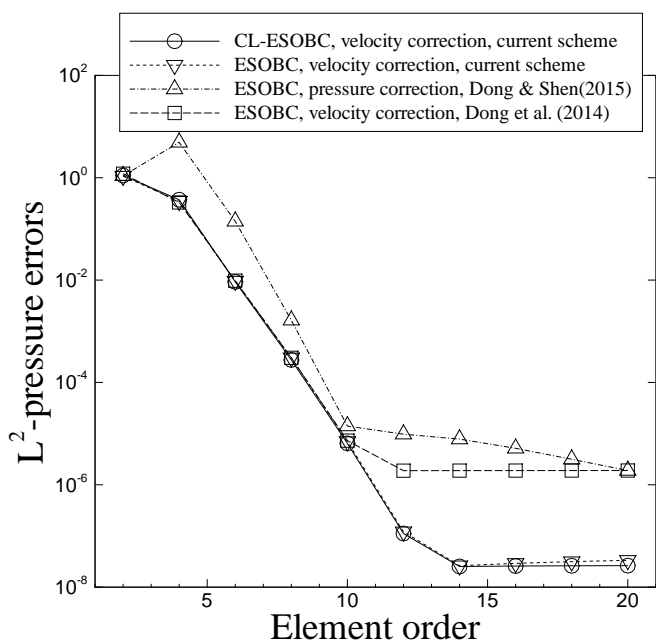

(a) (b)

Figure 2: Comparison between current and previous methods: $L^{2}$ errors of the $x$ velocity (a) and the pressure (b) as a function of the element order obtained with different methods. "CL-ESOBC" denotes the current convective-like energy-stable OBC (equation (4)). "ESOBC" denotes the energy-stable open boundary condition OBC-C of [18], i.e. equation (4) excluding the time-derivative term.

The above results suggest that for problems involving open boundaries the method presented in Section 2 exhibits an exponential convergence rate in space and a second-order convergence rate in time.

We next briefly compare the performance of the current method and those from $[14,18]$. While the algorithm of [18] is based on a pressure-correction strategy, both the current and the one from [14] are velocity-correction based schemes. The main differences between the current scheme and that of [14] have been pointed out in Section 2.2. In Figure 2 we compare the $L^{2}$ errors of the $x$ velocity component (plot (a)) and the pressure (plot (b)) as a function of the element order computed using different methods. These tests are conducted under the same conditions and with the same parameter values as those of Figure 1(b), but the open boundary condition and the numerical algorithm are changed. Using the current algorithm, we have acquired results with the current convective-like energy-stable open boundary condition (denoted by "CL-ESOBC" in plots), i.e. Equation (4), and also with the energy-stable open boundary condition OBC-C from [18] (denoted by "ESOBC" in plots), i.e. Equation (4) excluding the $\nu D_{0} \frac{\partial \mathbf{u}}{\partial t}$ term. Figures 2(a) and 2(b) have also included results with the boundary condition OBC-C computed using the algorithms of $[14,18]$. It is observed that the current scheme produces errors comparable to the previous methods at element orders prior to the error saturation. However, as the element order becomes large the error saturates at a much lower level (by one or two orders of magnitude) with the current algorithm than with those of [14, 18]. It can also be observed that the velocity-correction type schemes of the current work and [14] appear to result in generally lower pressure errors than the pressure-correction scheme of [18], as already pointed out by [18]. 

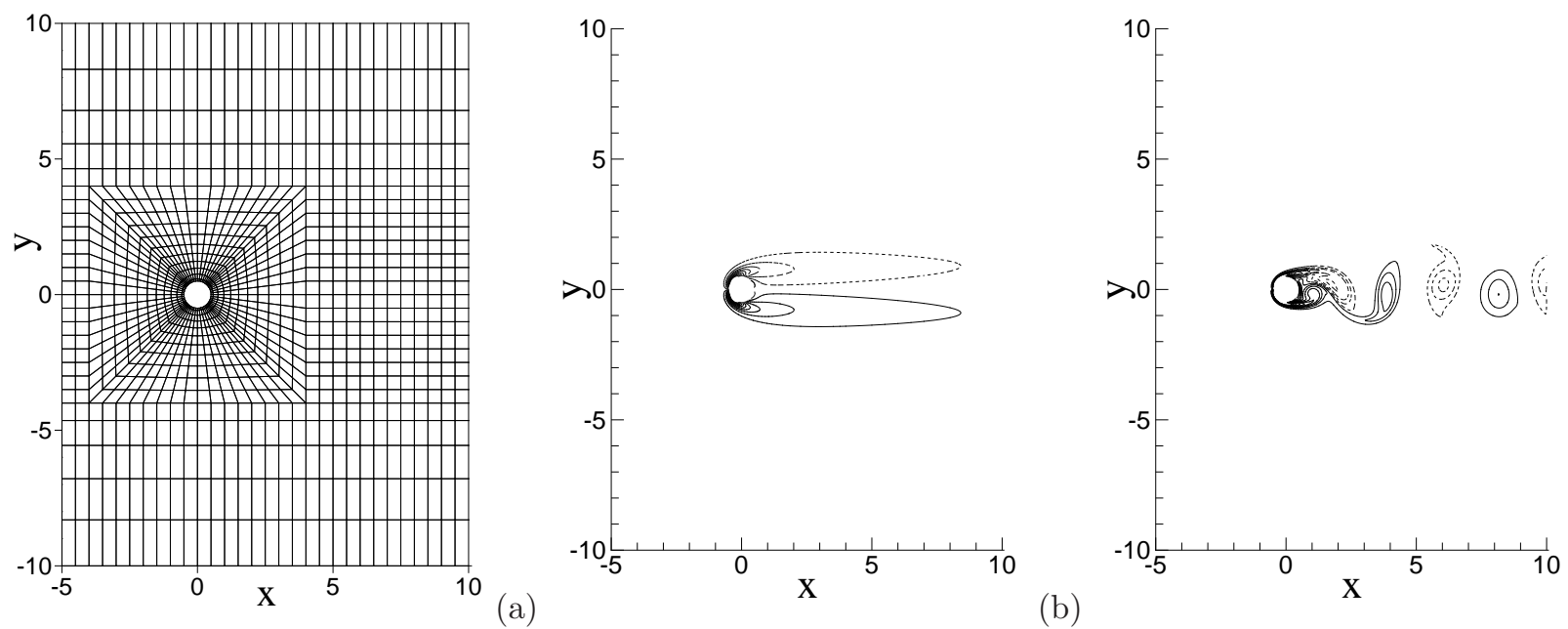

(b)

Figure 3: Cylinder flow: spectral element mesh (a), and contours of vorticity at Reynolds numbers $R e=30$ (b) and $R e=200$ (c). Dashed curves in (b) and (c) indicate negative vorticity values.

\subsection{Flow Past a Circular Cylinder}

In this subsection we consider a canonical wake problem, the flow past a circular cylinder, in two dimensions to test the performance of our method. The goal is to demonstrate the accuracy of the method by comparison with the experimental data, and to demonstrate its effectiveness in dealing with the backflow instability as the Reynolds number becomes large.

This flow problem has been used in [18] to test a different set of open boundary conditions and an associated pressure correction-based numerical algorithm. The flow configurations employed in the current work largely follow those of [18]. It should be noted that the open boundary condition and the algorithm being tested here are very different from those of [18].

Specifically, we consider a circular cylinder of diameter $d$, and a rectangular domain containing the cylinder, $-5 d \leqslant x \leqslant L$ and $-10 d \leqslant y \leqslant 10 d$, where $x=L$ is the right domain boundary to be specified below. The center of the cylinder coincides with the origin of the coordinate system. Four flow domains have been considered with different wake-region sizes. They respectively correspond to $L / d=5,10,15$ and 20, and are chosen in accordance with [18]. The flow domain with $L / d=10$ is illustrated in Figure $3($ a).

On the top and bottom domain boundaries $(y= \pm 10 d)$ we assume that the flow is periodic. So the configuration in actuality corresponds to the flow past an infinite array of cylinders aligned in the $y$ direction. On the left boundary $(x=-5 d)$ a uniform flow comes into the domain with a velocity $\mathbf{u}=(u, v)=\left(U_{0}, 0\right)$, where $U_{0}=1$ is the characteristic velocity scale. The right domain boundary $(x=L)$ is assumed to be open, where the fluid can freely move out of the domain and backflow may occur depending on the flow regime and the domain size. 
In order to simulate the problem, we discretize the domain using a mesh of quadrilateral spectral elements. Figure 3(a) shows the spectral element mesh for the domain with $L=10 d$, involving 1228 quadrilateral elements. The meshes for the domain sizes $L / d=15$ and 20 are generated based on the mesh in Figure 3(a), by duplicating the elements from the region $5 d \leqslant x \leqslant 10 d$ of Figure 3(a) and appending copies of them to the region $x \geqslant 10 d$. The mesh for the domain $L=5 d$ is generated by pruning the elements in the region $5 d \leqslant x \leqslant 10 d$ from Figure 3(a). The meshes for these three other domains respectively contain 968,1488 and 1748 quadrilateral elements.

In simulations we impose the periodic condition at $y / d= \pm 10$, and the velocity Dirichlet condition (3) at the inflow boundary $x=-5 d$ with a boundary velocity $\mathbf{w}=\left(U_{0}, 0\right)$. On the cylinder surface a velocity no-slip condition is imposed, i.e. the Dirichlet condition (3) with $\mathbf{w}=0$. At the open (outflow) boundary $x=L$ we impose the open boundary condition (4) with $\mathbf{f}_{b}=0$ and $\delta=\frac{1}{100}$.

We employ the algorithm developed in Section 2 to solve the incompressible Navier-Stokes equations. All the length variables are normalized by the cylinder diameter $d$ and the velocity is normalized by $U_{0}$. So the Reynolds number for this problem is defined by

$$
R e=\frac{1}{\nu}=\frac{U_{0} d}{\nu_{f}}
$$

where $\nu_{f}$ is the kinematic viscosity of the fluid. A range of Reynolds numbers (up to $R e=10000$ ) has been considered. We use an element order 6 for Reynolds numbers below 100, and an element order 8 for higher Reynolds numbers. For selected Reynolds numbers we have also performed simulations with even larger element orders (up to 12), and we observe that the difference in the results when compared with the element order 8 is small. The non-dimensional time step size is $U_{0} \Delta t / d=10^{-3}$ for Reynolds numbers below 100 and $U_{0} \Delta t / d=2.5 \times 10^{-4}$ for higher Reynolds numbers in the simulations.

As discussed in Section 2, the analogy between the open boundary condition (11) and the convective boundary condition (12) suggests that $\frac{1}{D_{0}}$ represents a convection velocity. For the majority of simulations in this section we employ the average velocity at the outflow boundary, $U_{0}$, as this convection velocity and set $D_{0}=\frac{1}{U_{0}}$ in the open boundary condition (4). This is the default $D_{0}$ value for the results reported in this section. For several selected Reynolds numbers we have also investigated the effects of $D_{0}$ on the simulation results. Results corresponding to the other $D_{0}$ values will be explicitly specified.

The cylinder wake can be classified into several regimes, exhibiting a variety of flow features. These have been expounded in the review paper [72]. For Reynolds numbers below about $R e=47$ the cylinder flow is two-dimensional and at a steady state. As the Reynolds number increases beyond this value, the cylinder wake becomes unsteady and is characterized by vortex sheddings. It remains two-dimensional for Reynolds numbers up to about $R e=180$. As the Reynolds number increases beyond $R e \approx 180$, the cylinder wake 


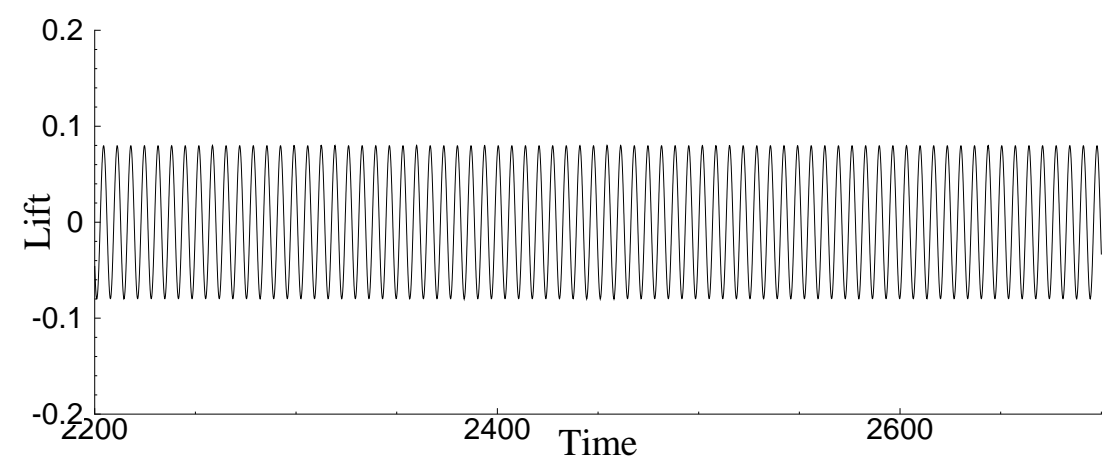

(a)

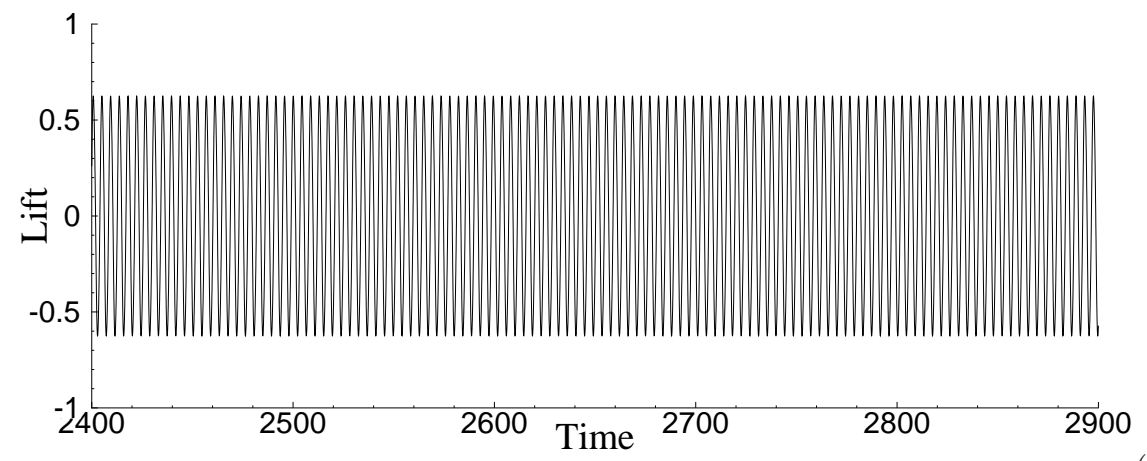

(b)

Figure 4: Cylinder flow: time histories of the lift force on the cylinder at Reynolds numbers (a) $R e=60$ and (b) $R e=500$. Results are obtained with $D_{0}=\frac{1}{U_{0}}$ in the open boundary condition.

develops an instability and the physical flow becomes three-dimensional. More complicated flow features and turbulence develop in the cylinder wake when the Reynolds number increases further. In Figures 3(b) and (c) we plot contours of the instantaneous vorticity obtained on the domain $L / d=10$ at Reynolds numbers $R e=30$ and $R e=200$, respectively. The results show a steady-state flow at $R e=30$ and regular vortex sheddings at $R e=200$.

We have computed and monitored the forces acting on the circular cylinder. In Figure 4 we show a window of the time histories of the lift (i.e. the force component in the cross-flow $y$ direction) at Reynolds numbers $R e=60$ and 500. The force signals exhibit quite regular fluctuations about a zero mean value at these Reynolds numbers.

Global flow parameters can be determined based on these force data. In Table 1 we have listed several flow parameters for two Reynolds numbers $R e=20$ and 100 obtained on different flow domains. They include: the drag coefficient $C_{d}=\frac{\bar{f}_{x}}{\frac{1}{2} \rho U_{0}^{2}}$, where $\bar{f}_{x}$ is the time averaged drag (i.e. force component in $\mathrm{x}$ direction) and $\rho=1$ is the fluid density; the root-mean-square (rms) drag coefficient $C_{d}^{\prime}=\frac{f_{x}^{\prime}}{\frac{1}{2} \rho U_{0}^{2}}$, where $f_{x}^{\prime}$ is the rms drag; and the rms lift coefficient $C_{L}=\frac{f_{y}^{\prime}}{\frac{1}{2} \rho U_{0}^{2}}$, where $f_{y}^{\prime}$ is the rms lift. These data indicate that the size of the wake region has an influence on the simulation results, and that as the wake region becomes 


\begin{tabular}{l|l|ccc}
\hline Reynolds number & Domain & $C_{d}$ & $C_{d}^{\prime}$ & $C_{L}$ \\
\hline 20 & $L / d=5$ & 2.294 & 0 & 0 \\
& $L / d=10$ & 2.317 & 0 & 0 \\
& $L / d=15$ & 2.317 & 0 & 0 \\
& $L / d=20$ & 2.317 & 0 & 0 \\
\hline 100 & $L / d=5$ & 1.441 & $8.491 E-3$ & 0.261 \\
& $L / d=10$ & 1.459 & $7.631 E-3$ & 0.254 \\
& $L / d=15$ & 1.462 & $7.700 E-3$ & 0.253 \\
& $L / d=20$ & 1.462 & $7.714 E-3$ & 0.253 \\
\hline
\end{tabular}

Table 1: Cylinder flow: effect of the domain size on the global flow parameters. $C_{d}$ : drag coefficient or time-averaged mean drag coefficient; $C_{d}^{\prime}$ : rms drag coefficient; $C_{L}$ : rms lift coefficient.
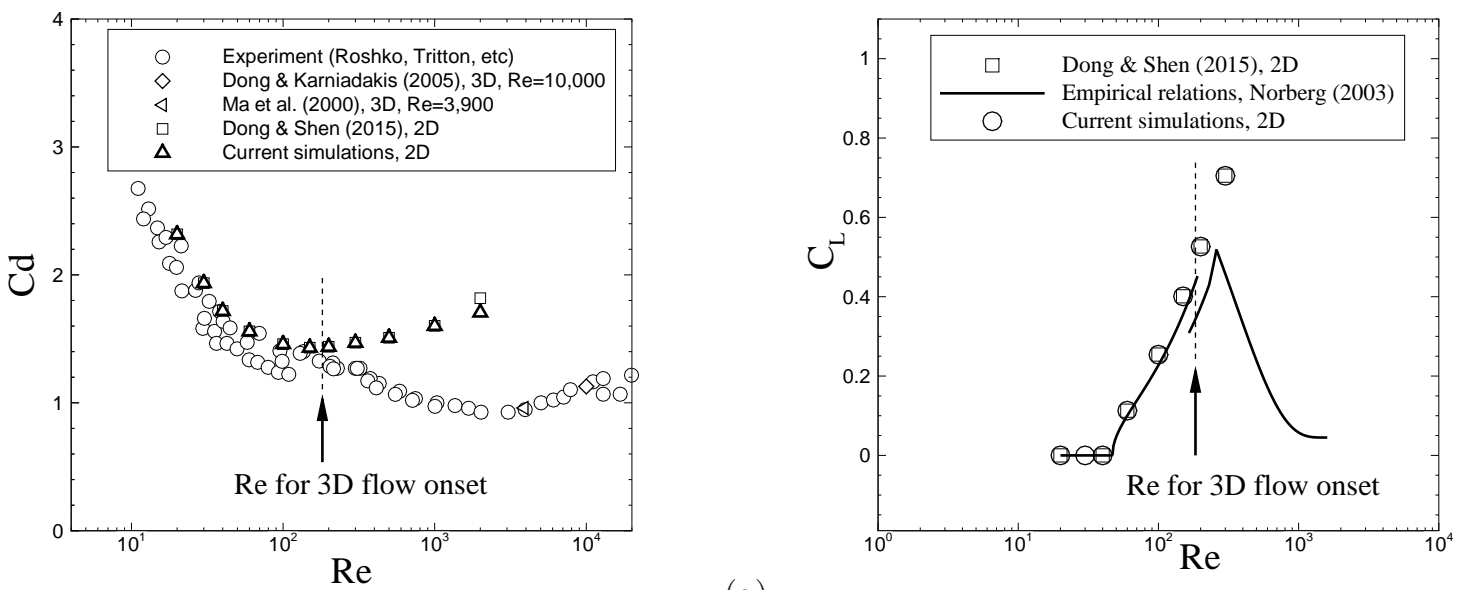

(a)

Figure 5: Cylinder flow: Comparison of (a) drag coefficient and (b) rms lift coefficient versus Reynolds number between the current simulations, the experimental data, and the simulation results of [18].

sufficiently large the flow parameters computed from the simulations remain essentially unchanged as the domain size further increases. It can be observed that the flow domain $L / d=10$ is very close to the point where further increase in the domain size no longer results in significant changes in the results. In light of this observation, our subsequent discussions will be mainly based on the results obtained on the domain $L / d=10$.

To demonstrate the accuracy of the method, we compare the force parameters computed from the current simulations with those from the experimental measurements and from other simulations in the literature. In Figure 5(a) we plot the drag coefficient $\left(C_{d}\right)$ as a function of Reynolds number from the current simulations, from a number of experiments $[71,9,21,69,61]$, and from the simulations of $[48,13,18]$. Note that the simulations in [18] and in the current work are both two-dimensional, while those of $[48,13,15]$ are threedimensional. The current results correspond to the domain size $L / d=10$ and $D_{0}=\frac{1}{U_{0}}$ in the open boundary condition (4). They agree with those of [18] very well. Note that both the numerical algorithm and the 


\begin{tabular}{lcc}
\hline Reference & $R e=100$ & $R e=200$ \\
Braza et al. (1986) [5] & 0.21 & 0.55 \\
Engelman \& Jamnia (1990) [19] & 0.26 & - \\
Meneghini \& Bearman (1993) [49] & - & 0.54 \\
Beaudan \& Moin (1994) [2] & 0.24 & - \\
Zhang et al. (1995) [73] & 0.25 & 0.53 \\
Tang \& Audry (1997) [66] & 0.21 & 0.45 \\
Persillon \& Braza (1998) [58] & 0.27 & 0.56 \\
Zhang \& Dalton (1998) [74] & - & 0.48 \\
Kravchenko et al. (1999) [43] & 0.22 & - \\
Hwang \& Lin (1992) [35] & 0.27 & 0.42 \\
Franke et al. (1990) [24] & - & 0.46 \\
Karniadakis (1988) [39] & - & 0.48 \\
Newman \& Karniadakis (1995) [51] & - & 0.51 \\
Newman \& Karniadakis (1996) [52] & 0.24 & - \\
Dong \& Shen (2010) [16] & - & 0.501 \\
Dong \& Shen (2015) [18] & 0.254 & 0.527 \\
Current simulation (domain $L / d=10)$ & 0.254 & 0.526 \\
Current simulation (domain $L / d=20)$ & 0.253 & - \\
\hline
\end{tabular}

Table 2: Cylinder flow: Comparison of rms lift coefficients at $R e=100$ and $R e=200$ between current simulations and other simulations from literature.

outflow boundary condition in the current work are different from those of [18]. In the two-dimensional regime the current results also agree well with the experimental data. But for Reynolds numbers where the physical flow has become three-dimensional (beyond about $R e=180$ ), the current two-dimensional simulations result in overly large drag coefficients compared to the experiments, and the discrepancy grows with increasing Reynolds number.

Figure 5(b) is a comparison of the rms lift coefficient $C_{L}$ as a function of the Reynolds number between current simulations, the experiment of [53], and the simulations of [18]. The curves show the empirical relation given by [53] based on several experimental sources, which exhibits a hysteresis around the Reynolds numbers where the two-dimensional to three-dimensional flow transition occurs. The lift coefficients from the current simulations and from [18] agree with each other almost exactly. In the two-dimensional regime the current results agree with the empirical relation from [53] reasonably well. In the three-dimensional regime, however, the current two-dimensional simulations grossly over-predict the lift coefficient, which is a well-known phenomenon about two-dimensional simulations (see e.g. [13, 15]).

In Table 2 we have summarized the rms lift coefficients $\left(C_{L}\right)$ for Reynolds numbers $R e=100$ and 200 from a number of two-dimensional simulations in the literature. We have also listed the $C_{L}$ values on flow domains with $L / d=10$ and 20 from the current simulations for comparison; see Table 1 for $C_{L}$ values on the other domains at $R e=100$. One can observe a spread in the $C_{L}$ values from different simulations. The lift coefficients from the current work are well within the range of values from the literature. 


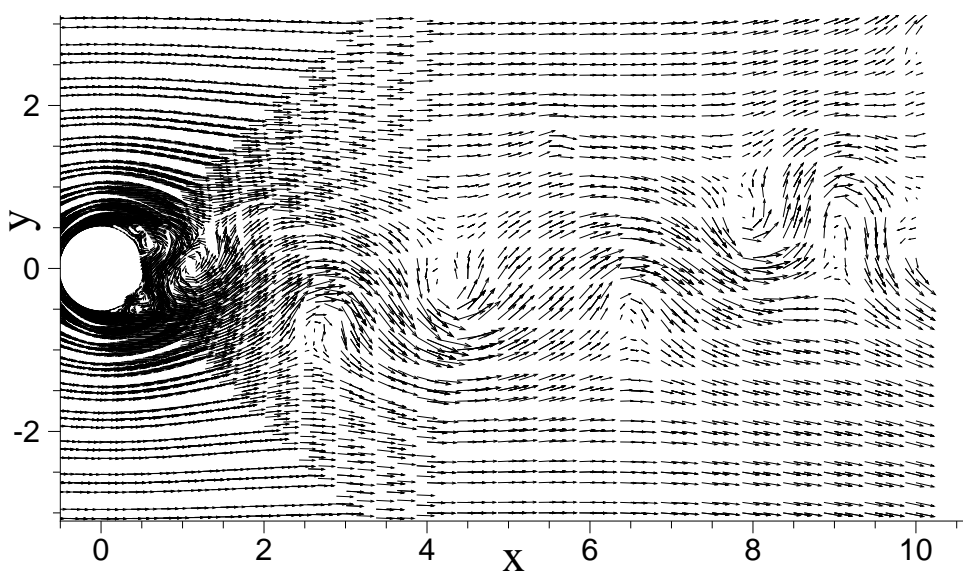

(a)

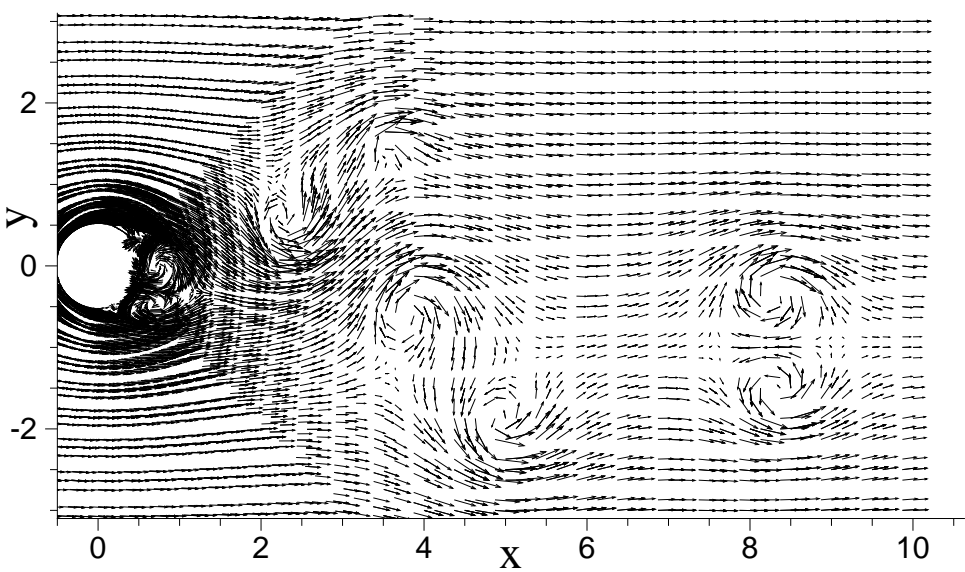

(b)

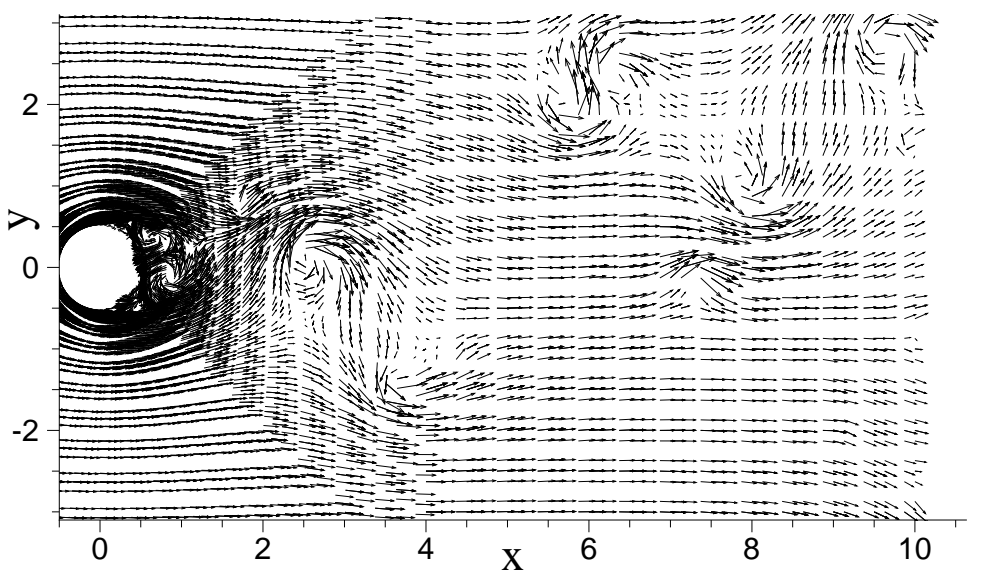

(c)

Figure 6: Cylinder flow: snapshots of instantaneous velocity fields at Reynolds numbers (a) $R e=2000$, (b) $R e=5000$, and (c) $R e=10000$. Velocity vectors are plotted on every fifth quadrature points in each direction within each element. 


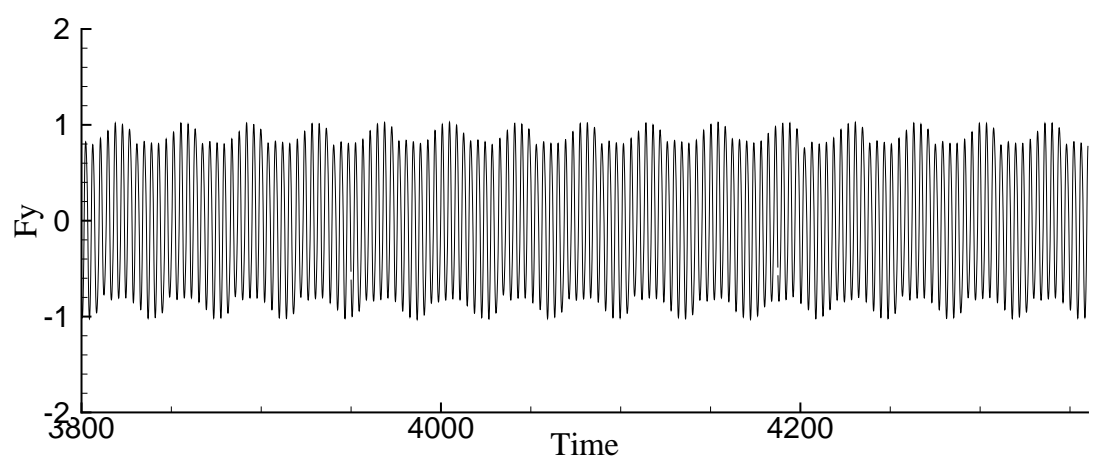

(a)

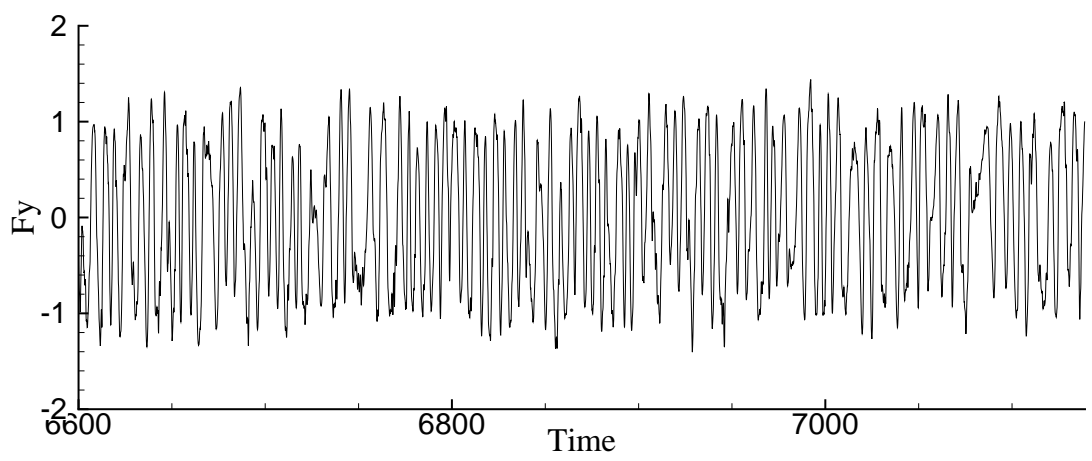

(b)

Figure 7: Time histories of the lift force on the cylinder at Reynolds numbers (a) $R e=2000$, and (b) $R e=10000$.

Let us next look into the effectiveness of the open boundary condition and the algorithm from Section 2 for dealing with the backflow instability. We will consider the cylinder flow at higher Reynolds numbers, ranging from $R e=2000$ to $R e=10000$. At these Reynolds numbers energetic vortices are observed to pass through the outflow boundary and induce strong backflows in that region. This creates a severe instability issue, and makes the simulation immensely challenging.

Thanks to the energy stability, the current open boundary condition provides an effective way for overcoming this instability. In Figure 6 we show distributions of the instantaneous velocity at three Reynolds numbers $R e=2000,5000$ and 10000 . The results are obtained on the domain $L / d=10$, with $D_{0}=\frac{1}{U_{0}}$ in the open boundary condition (4). Energetic vortices can be clearly observed at the open boundary (see e.g. Figure 6(c)).

We have performed long-time simulations at these Reynolds numbers using the current method. The long-term stability of the simulations is demonstrated by Figure 7, in which we show a window of the time histories (over 30 flow-through time) of the lift force on the cylinder at Reynolds numbers $R e=2000$ and $R e=10000$ obtained on the domain $L / d=10$. At $R e=2000$ the lift signal exhibits a modulation in its amplitude. At $R e=10000$ the fluctuation becomes quite chaotic and the vortex-shedding frequency appears 
to vary over time. These results show that simulations using the current method are long-term stable in the presence of strong vortices and backflows at the outflow/open boundaries.

In contrast, the boundary condition (10) is observed to be unstable at these Reynolds numbers considered here. The computation instantly blows up as the vortices hit the outflow/open boundary.

We observe that the open boundary condition developed herein allows the vortices to discharge from the domain in a fairly natural fashion, even at quite high Reynolds numbers. This is illustrated by Figure 8, in which a temporal sequence of snapshots of the velocity fields at $R e=10000$ have been shown. These velocity fields illustrate the process in which the vortex originally located near the outflow boundary and the wake centerline in Figure 8(a) is discharged from the domain through the outflow boundary. These results are obtained using the open boundary condition (4) with $D_{0}=\frac{1}{U_{0}}$. As the vortex core crosses the outflow boundary (Figure 8(b)), one can observe a region of backflows (Figure 8(b)-(c)). Some distortion to the vortex can be observed, especially after the vortex core passes through the outflow boundary (Figure 8(d)). Overall, the current method appears to allow the vortex to cross the outflow boundary and exit the domain in an approximately natural way (Figures $8(\mathrm{~b})-(\mathrm{h})$ ) even at such a substantial Reynolds number.

Let us next look into the effects of the $D_{0}$ value in the open boundary condition (4) on the simulation results. By an analogy between the current open boundary condition and the usual convective boundary condition (12), we observe that $\frac{1}{D_{0}}$ should correspond to a convection velocity scale $U_{c}$ at the outflow boundary, i.e. $\frac{1}{D_{0}}=U_{c}$, as is discussed in Section 2. The simulation results for the cylinder flow presented so far are obtained with a value $\frac{1}{D_{0}}=U_{c}=U_{0}$, where $U_{0}$ is the average velocity at the outflow boundary.

\begin{tabular}{l|l|ccc}
\hline Reynolds number & $D_{0} U_{0}$ & $C_{d}$ & $C_{d}^{\prime}$ & $C_{L}$ \\
\hline 20 & 0.0 & 2.317 & 0.0 & 0.0 \\
& 0.5 & 2.317 & 0.0 & 0.0 \\
& 1.0 & 2.317 & 0.0 & 0.0 \\
& 2.0 & 2.317 & 0.0 & 0.0 \\
\hline 100 & 5.0 & 2.317 & 0.0 & 0.0 \\
\hline 10000 & 0.0 & 1.459 & $7.627 E-3$ & 0.254 \\
& 0.5 & 1.459 & $7.630 E-3$ & 0.254 \\
& 1.0 & 1.459 & $7.631 E-3$ & 0.254 \\
& 2.0 & 1.459 & $7.639 E-3$ & 0.254 \\
& 5.0 & 1.459 & $7.656 E-3$ & 0.254 \\
\hline & 0.0 & 1.862 & 0.449 & 1.483 \\
& 0.5 & 1.881 & 0.421 & 1.506 \\
& 1.0 & 1.843 & 0.442 & 1.474 \\
& 2.0 & 1.893 & 0.432 & 1.504 \\
& 5.0 & 1.858 & 0.446 & 1.474 \\
\hline
\end{tabular}

Table 3: Effect of $D_{0}$ in OBC on global flow parameters: drag and lift coefficients for cylinder flow obtained with different $D_{0}$ values. $C_{d}$ : drag coefficient or time-averaged mean drag coefficient; $C_{d}^{\prime}$ : root-mean-square (rms) drag coefficient; $C_{L}$ : rms lift coefficient. 

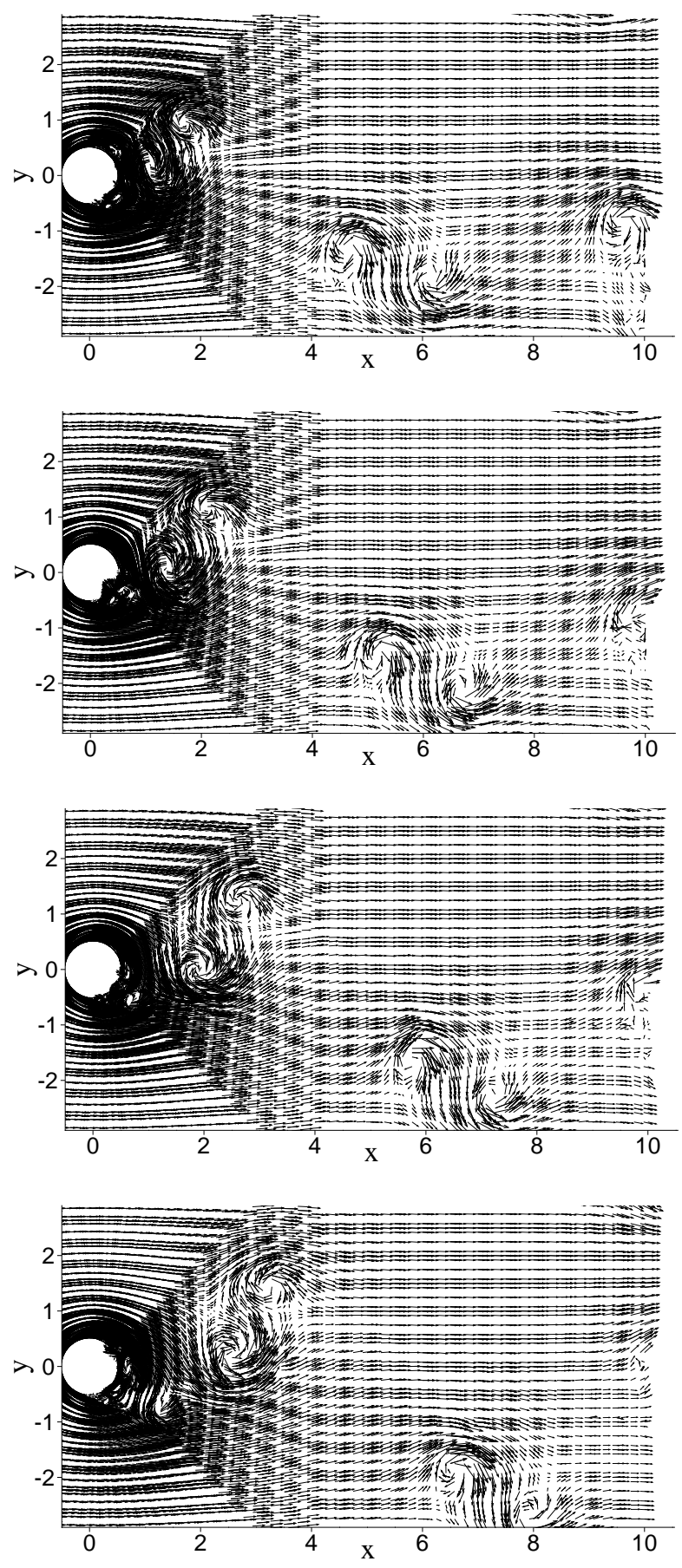

(g)

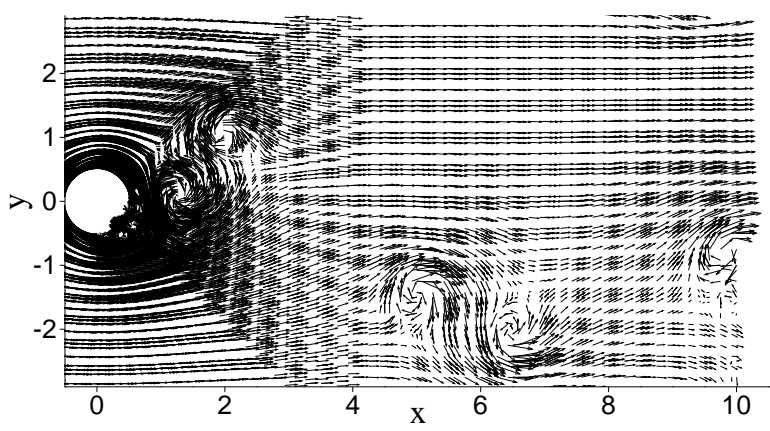

(b)

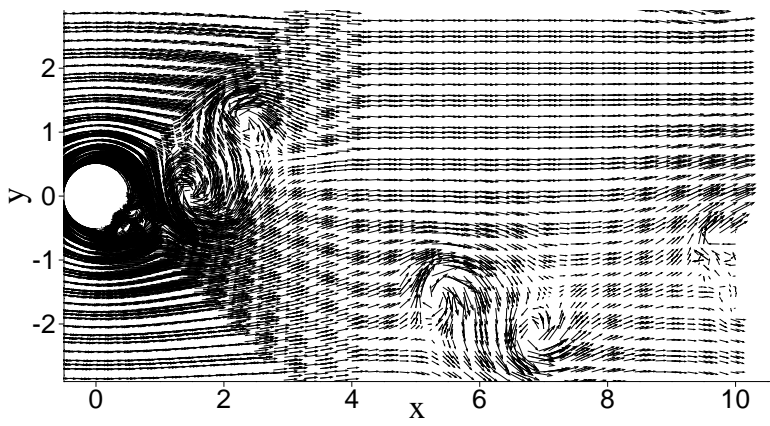

(d)

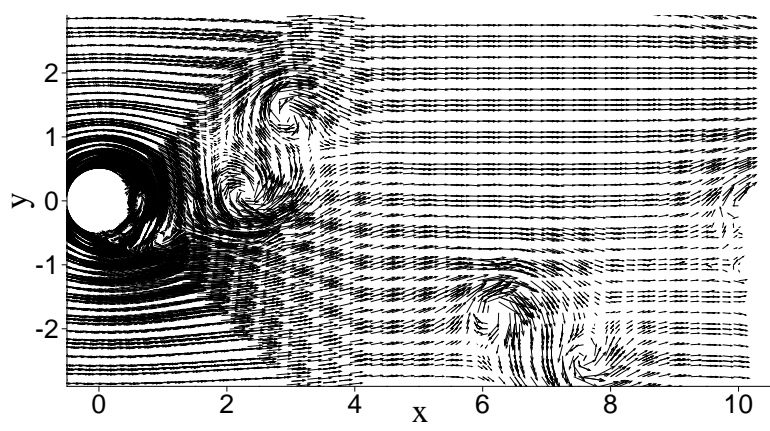

(f)

(h)

Figure 8: Discharge of vortices away from domain $(R e=10000)$ : temporal sequence of snapshots of velocity fields at (a) $t=6912.5$, (b) $t=6913$, (c) $t=6913.25$, (d) $t=6913.5$, (e) $t=6914$, (f) $t=6914.5$, (g) $t=6915$, (h) $t=6915.5$. Velocity vectors are plotted on every fifth quadrature points in each direction within each element. $D_{0}=\frac{1}{U_{0}}$ in the open boundary condition. 


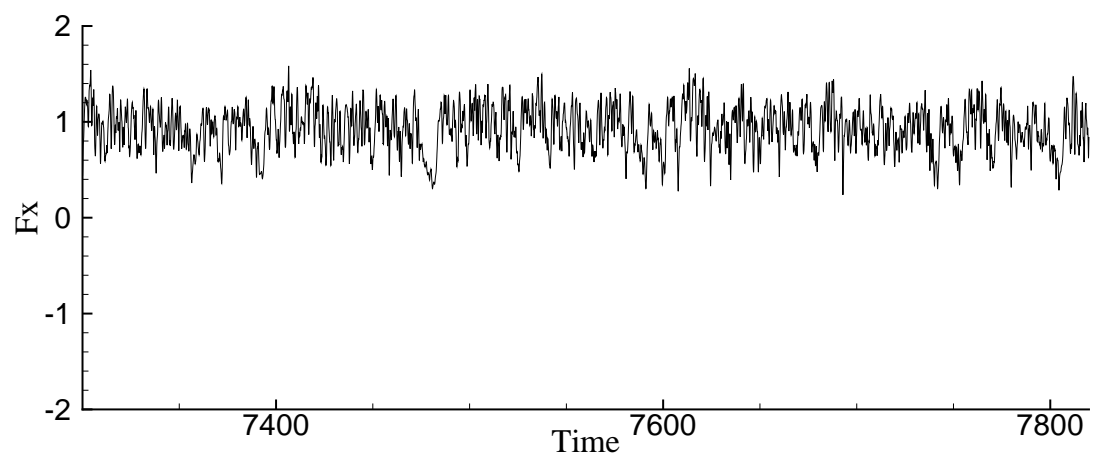

(a)

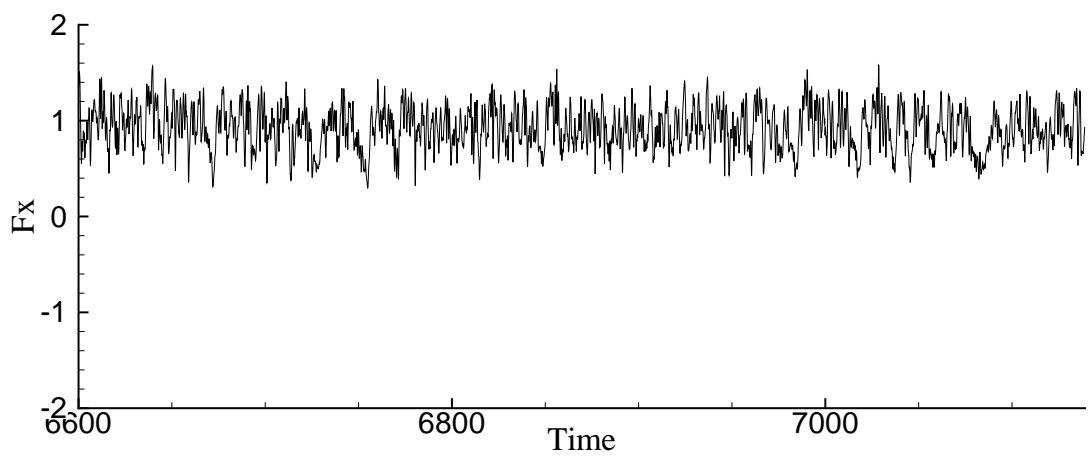

(b)

Figure 9: Time histories of the drag on the cylinder at $R e=10000$ obtained using different $D_{0}$ values in OBC: (a) $D_{0}=0$, and (b) $D_{0}=\frac{1}{U_{0}}$.

We have observed that the variation in the $D_{0}$ value in the open boundary condition (4) has little or essentially no effect on the global physical quantities such as the forces on the cylinder. This is illustrated in Figure 9 with the time histories of the drag at Reynolds number $R e=10000$ corresponding to two values $D_{0} U_{0}=0$ ( or $U_{c}=\infty$ ) and $D_{0} U_{0}=1$ (or $U_{c}=U_{0}$ ) in the open boundary condition (4). The two drag signals exhibit qualitatively similar characteristics. A quantitative comparison of the global physical quantities corresponding to several $D_{0}$ values is given in Table 3 . The table includes the data for the mean drag coefficient $\left(C_{d}\right)$, rms drag coefficient $\left(C_{d}^{\prime}\right)$, and the rms lift coefficient $\left(C_{L}\right)$ for Reynolds numbers $R e=20,100$ and 10000 on the flow domain with $L=10 d$. We have considered several $D_{0}$ values in these tests, corresponding to $D_{0} U_{0}=0,0.5,1,2$ and 5 , or equivalently $U_{c}=\infty, 2 U_{0}, U_{0}, \frac{U_{0}}{2}$ and $\frac{U_{0}}{5}$. One can observe that at $R e=20$ and $R e=100$ these global physical quantities obtained using these several $D_{0}$ values are exactly or almost exactly the same. At $R e=10000$, they are also close for different $D_{0}$ values, with a maximum difference of about $2.7 \%$ for $C_{d}$, about $6.7 \%$ for $C_{d}^{\prime}$, and about $2.1 \%$ for $C_{L}$. These results suggest that the value of $D_{0}$ in the open boundary condition (4) has little effect quantitatively on the physical quantities of the flow.

The main effect of $D_{0}$ appears to be on the qualitative features of the flow, such as the smoothness of 


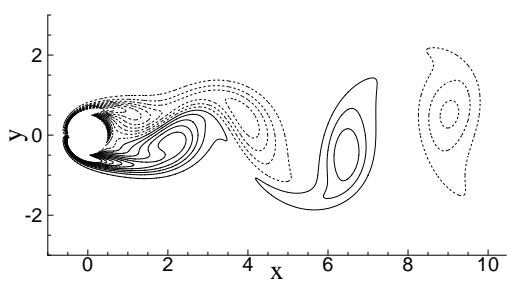

(a)

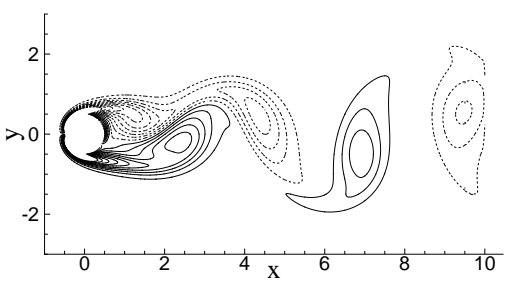

(b)
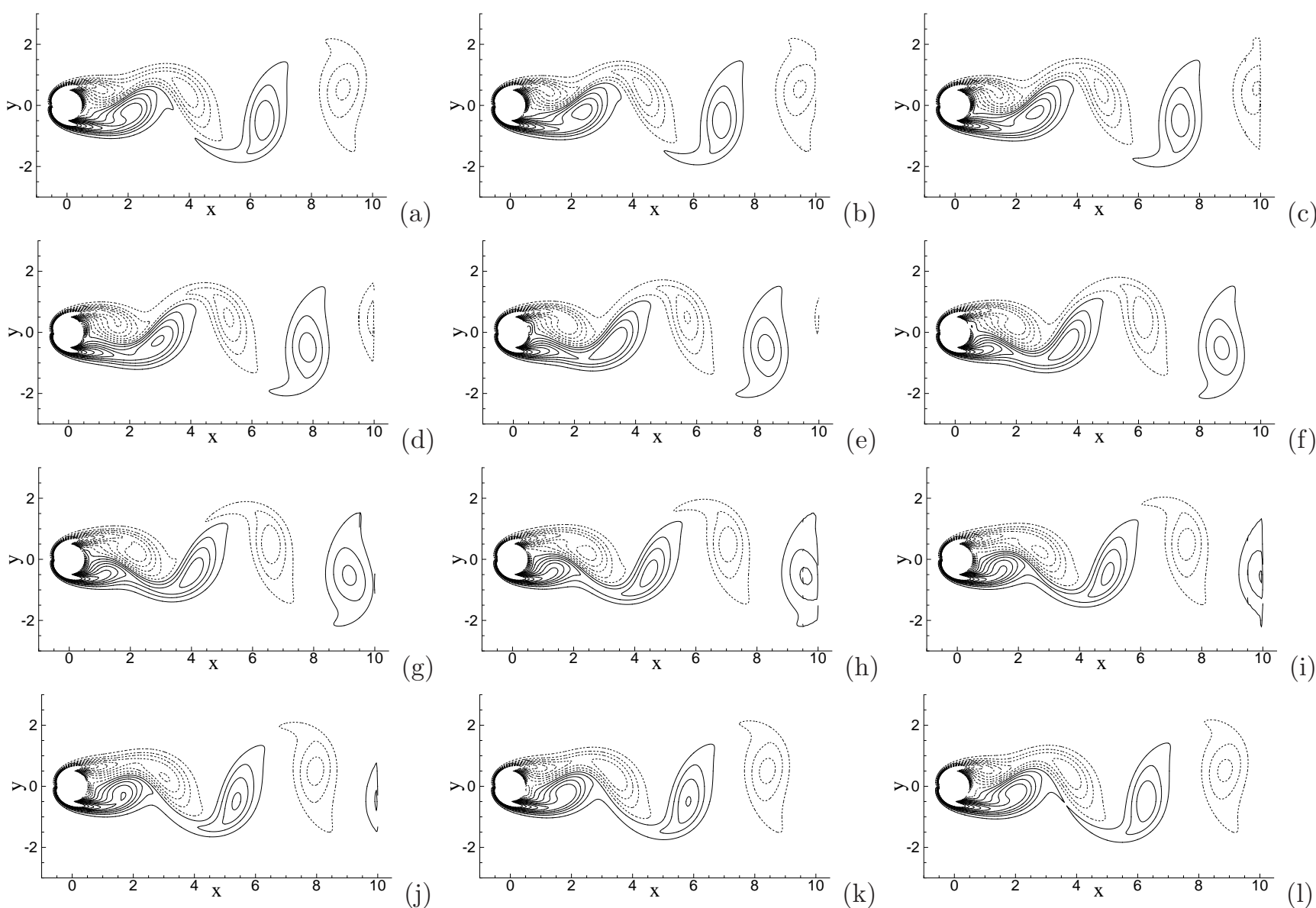

h)

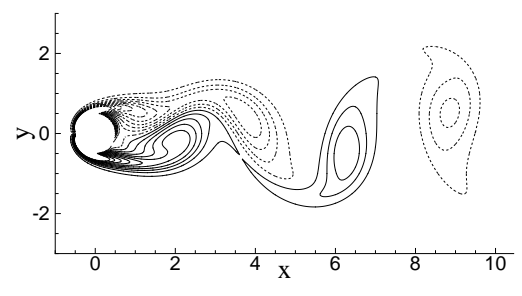

Figure 10: Cylinder flow at $R e=100$ : Temporal sequence of snapshots of instantaneous vorticity at (a) $t=3939,(\mathrm{~b}) t=3939.5,(\mathrm{c}) t=3940$, (d) $t=3940.5,(\mathrm{e}) t=3941$, (f) $t=3941.5$, (g) $t=3942,(\mathrm{~h})$ $t=3942.5$, (i) $t=3943$, (j) $t=3943.5$, (k) $t=3944$, (l) $t=3944.5$. Results are obtained using $D_{0}=0$ in the open boundary condition. Dashed curves denote negative vorticity values.

the velocity field, and on the velocity derivatives in regions local to the outflow boundary. We observe that the current open boundary condition (4) with appropriate $D_{0}>0$ result in smoother velocity distributions at the outflow boundary and can allow the vortices to cross the outflow boundary more smoothly and in a more natural way when compared to the boundary condition with $D_{0}=0$, which corresponds exactly to the boundary condition OBC-C studied in [18]. To demonstrate this point, we show in Figure 10 a temporal sequence of snapshots of the instantaneous vorticity covering approximately one period of vortex shedding at Reynolds number $R e=100$, obtained with $D_{0}=0$ in the open boundary condition. For comparison, Figure 11 shows another temporal sequence of snapshots of the instantaneous vorticity at $R e=100$ obtained using $D_{0}=\frac{1}{U_{0}}$ in the open boundary condition. Note that at this Reynolds number no backflow occurs at the outflow boundary, and therefore the term involving $\Theta_{0}$ in the boundary condition (4) disappears and has no effect. With $D_{0}=0$, as the vortex passes through the outflow boundary, one can observe a more pronounced distortion to the vorticity contours in regions near the boundary; see e.g. Figures 10(b)-(d), 


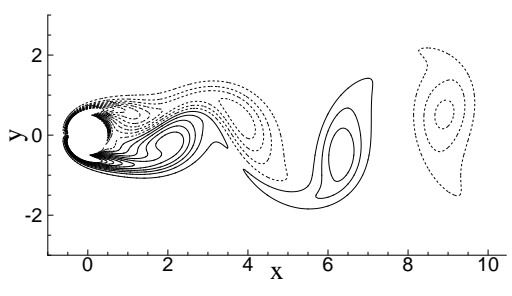

(a)

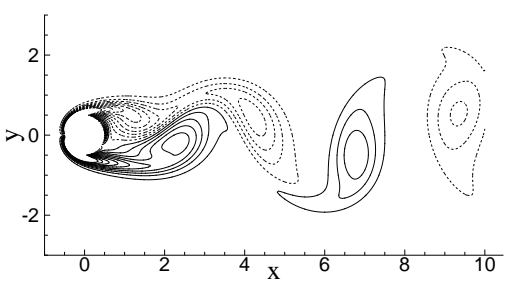

(b)
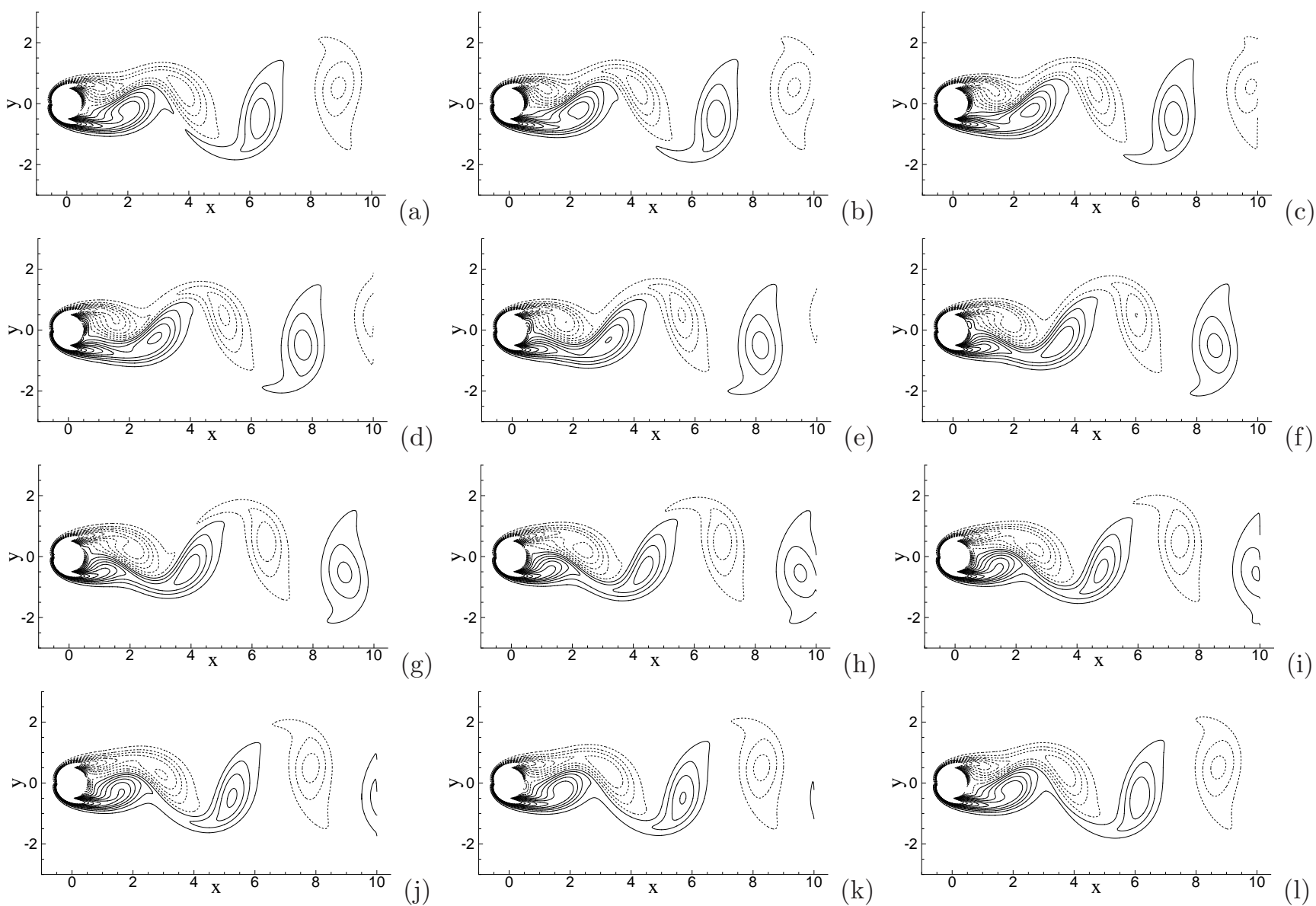

Figure 11: Cylinder flow at $R e=100$ : Temporal sequence of snapshots of instantaneous vorticity at (a) $t=2867.5$, (b) $t=2868$, (c) $t=2868.5$, (d) $t=2869$, (e) $t=2869.5$, (f) $t=2870$, (g) $t=2870.5$, (h) $t=2871$, (i) $t=2871.5$ (j) $t=2872$, (k) $t=2872.5$, (l) $t=2873$. Results are obtained using $D_{0}=\frac{1}{U_{0}}$ in the open boundary condition. Dashed curves denote negative vorticity values.

and 10(h)-(j). In contrast, with $D_{0}=\frac{1}{U_{0}}$, the vortices appear to exit the domain in a more natural fashion and there appears to be notably less distortion to the vorticity contours as the vortex passes through the outflow boundary; see e.g. Figures 11(b)-(d), and 11(h)-(j). The vorticity patterns at $R e=100$ resulting from other $D_{0}$ values have also been studied. It is observed that the results with $D_{0} U_{0}=0.5$ (i.e. $U_{c}=2 U_{0}$ ) and $D_{0} U_{0}=2$ (i.e. $U_{c}=\frac{U_{0}}{2}$ ) are comparable to, but slightly worse than, that with $D_{0}=\frac{1}{U_{0}}$ in terms of the distortion to the vorticity patterns as the vortex crosses the outflow boundary. The result with $D_{0} U_{0}=5$ (i.e. $\left.U_{c}=U_{0} / 5\right)$ is comparable to, but appears somewhat worse than, that with $D_{0}=0$ (i.e. $U_{c}=\infty$ ).

To provide a quantitative sense of how $D_{0}$ affects the flow field at the outflow boundary, we look into the integrals of the energy and the vorticity-squared over several cross-stream planes at or near the outflow boundary,

$$
E_{g}(t)=\int_{A} \frac{1}{2}|\mathbf{u}|^{2} d A, \quad \Psi(t)=\int_{A}|\boldsymbol{\omega}|^{2} d A
$$



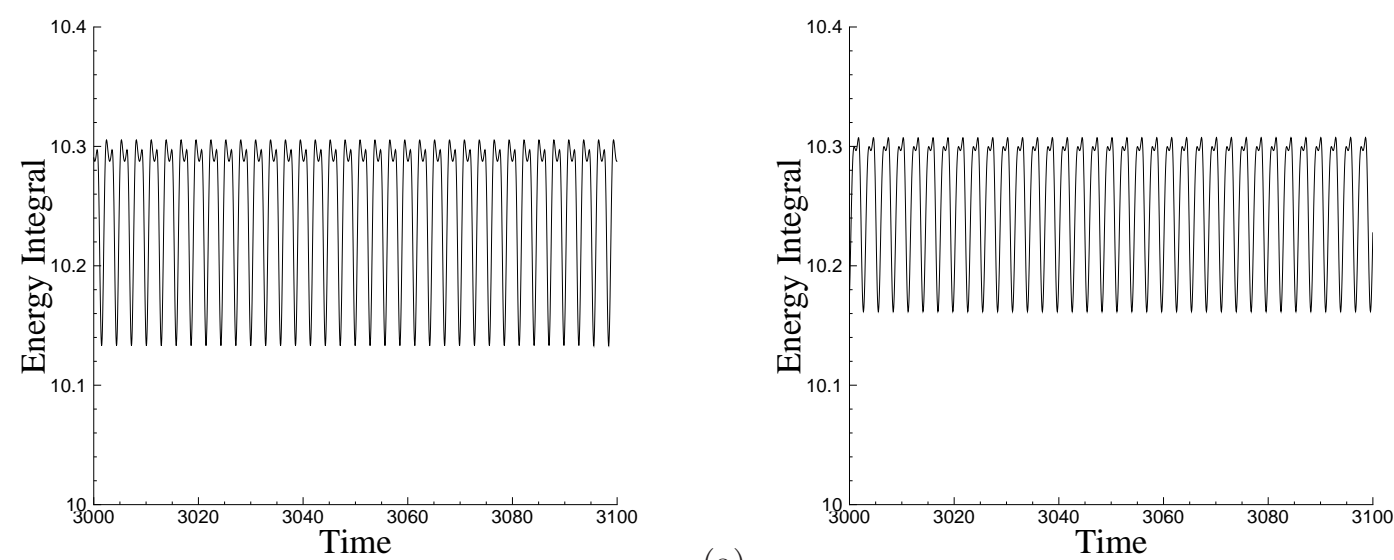

(a)
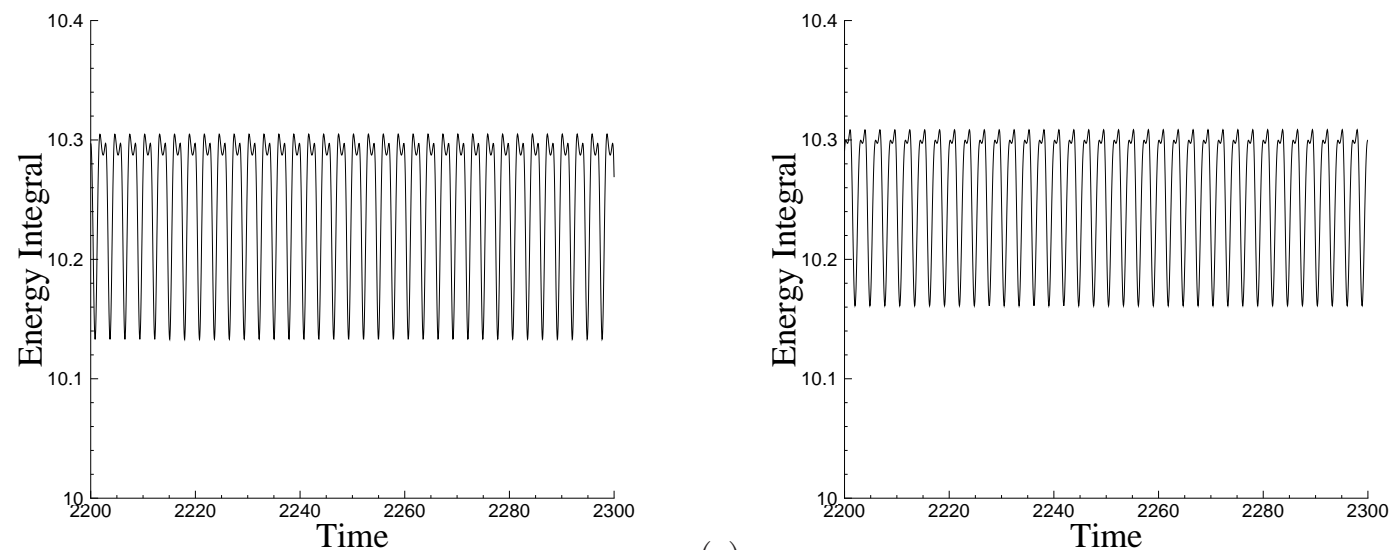

(c)

Figure 12: Cylinder flow $(R e=100)$ : Evolution of the energy integral $E_{g}(t)$ over a cross-stream plane at $x=9 d$ (plots (a) and (c)) and at the outflow boundary $x=10 d$ (plots (b) and (d)). Results of (a) and (b) are obtained using the open boundary condition with $D_{0}=0$, and those of (c) and (d) correspond to $D_{0}=\frac{1}{U_{0}}$.

where $\boldsymbol{\omega}$ is the vorticity, and $A$ represents some cross-stream plane (i.e. fixed $x$ coordinate). We consider three cross-stream planes: the outflow plane $(x=10 d)$, and two nearby interior planes located at $x=9 d$ and $9.5 d$. It is expected that $E_{g}(t)$ and $\Psi(t)$ would decay along the downstream direction due to viscous dissipation, and that at the outflow plane these quantities would be close to at the nearby interior planes if $R e$ is not too small. Figure 12 shows a window of the time histories of $E_{g}(t)$ at the outflow plane and the interior plane $x=9 d$ for $R e=100$, corresponding to the open boundary condition with $D_{0}=0$ (Figures $12(\mathrm{a})-(\mathrm{b}))$ and $D_{0}=\frac{1}{U_{0}}$ (Figures $\left.12(\mathrm{c})-(\mathrm{d})\right)$. The energy integral is observed to fluctuate in time, and the fluctuation amplitude at the outflow plane is comparable but a little smaller than at the interior plane $x=9 d$. The results with $D_{0}=0$ and $D_{0}=\frac{1}{U_{0}}$ appear very close to each other at both planes.

The vorticity-squared integral (or enstrophy) $\Psi(t)$, on the other hand, exhibits dramatic differences. 

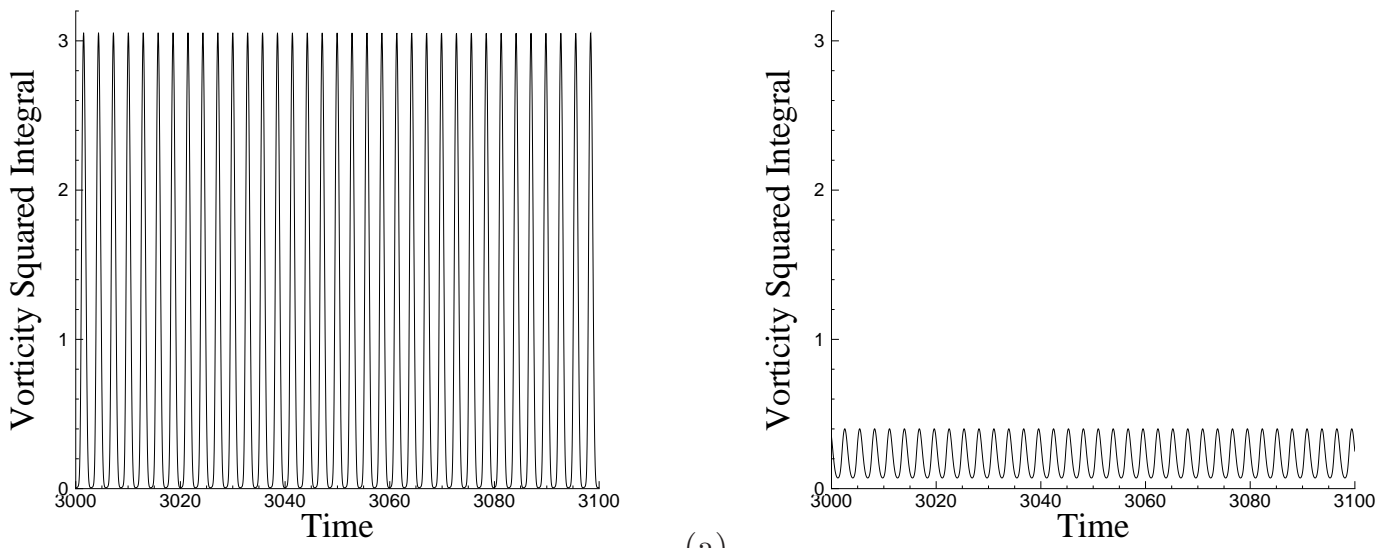

(a)
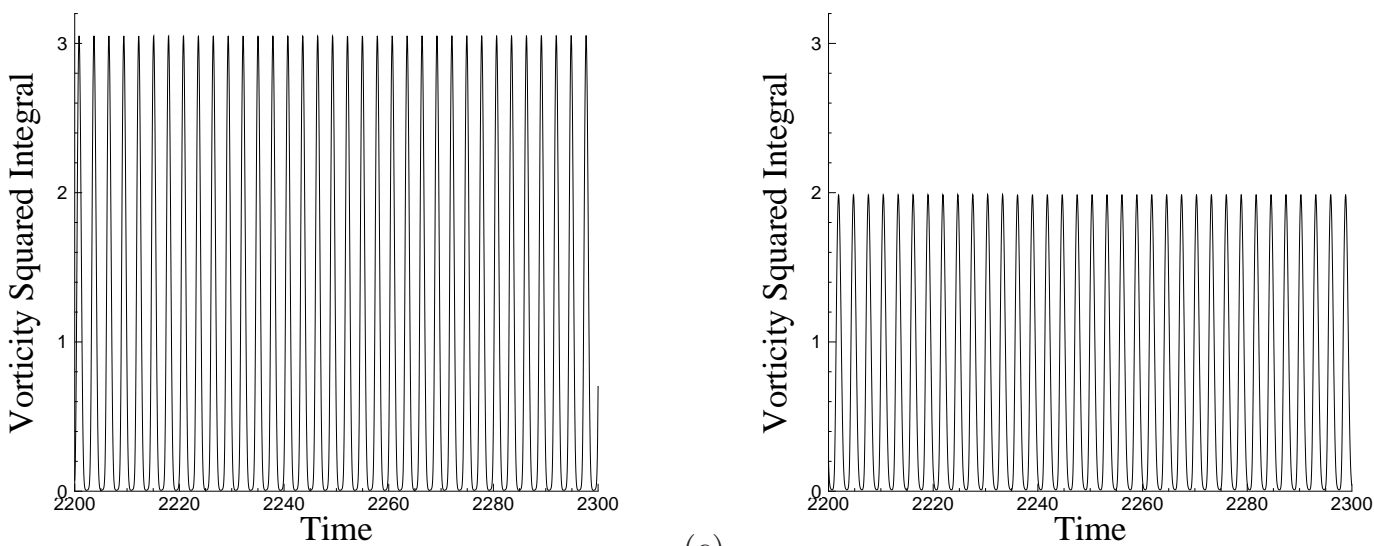

(c)

Figure 13: Cylinder flow (Re $=100)$ : Evolution of the vorticity-squared integral $\Psi(t)$ over a cross-stream plane at $x=9 d$ (plots (a) and (c)) and the outflow plane $x=10 d$ (plots (b) and (d)). Results in (a) and (b) are obtained using the open boundary condition with $D_{0}=0$, and those of (c) and (d) correspond to $D_{0}=\frac{1}{U_{0}}$.

Figure 13 shows a window of time histories of $\Psi(t)$ at the outflow plane and the interior plane $x=9 d$ corresponding to $D_{0}=0$ and $D_{0}=\frac{1}{U_{0}}$ in the open boundary condition. Comparison between Figures 13(a) and 13(c) indicates that at the plane $x=9 d$ the vorticity characteristics obtained with $D_{0}=0$ and $D_{0}=\frac{1}{U_{0}}$ are comparable. However, at the outflow plane the results are significantly different with $D_{0}=0$ and $D_{0}=\frac{1}{U_{0}}$, as shown by Figures $13(\mathrm{~b})$ and (d). The vorticity at the outflow plane resulting from $D_{0}=0$ is substantially smaller in amplitude than that from $D_{0}=\frac{1}{U_{0}}$. Comparison between Figures $13(\mathrm{a})$ and (b) shows a drastic decay in the vorticity between the plane $x=9 d$ and the outflow plane $x=10 d$ when using the open boundary condition with $D_{0}=0$. One can also observe a decay in the vorticity with $D_{0}=\frac{1}{U_{0}}$, but it is much less substantial.

The drastic difference in enstrophy between the outflow plane and the nearby interior plane with $D_{0}=0$ is 


\begin{tabular}{l|l|ccccc}
\hline & $D_{0} U_{0}$ & 0.0 & 0.5 & 1.0 & 2.0 & 5.0 \\
\hline Plane $x=9 d$ & $\bar{\Psi}$ & 0.957 & 0.956 & 0.956 & 0.957 & 0.957 \\
& $\Psi^{\prime}$ & 1.104 & 1.104 & 1.104 & 1.104 & 1.104 \\
\hline Plane $x=9.5 d$ & $\bar{\Psi}$ & 0.946 & 0.940 & 0.934 & 0.923 & 0.895 \\
& $\Psi^{\prime}$ & 1.033 & 1.027 & 1.021 & 1.010 & 0.984 \\
\hline Outflow plane & $\bar{\Psi}$ & 0.210 & 0.390 & 0.724 & 1.857 & 8.929 \\
$(x=10 d)$ & $\Psi^{\prime}$ & 0.119 & 0.346 & 0.728 & 1.956 & 9.261 \\
\hline
\end{tabular}

Table 4: Cylinder flow $(R e=100)$ : Mean $(\bar{\Psi})$ and $\operatorname{rms}\left(\Psi^{\prime}\right)$ of the vorticity-squared integral $\Psi(t)$ on the outflow plane $(x=10 d)$ and two cross-stream planes near the outflow boundary $(x=9 d$ and $9.5 d)$ corresponding to the open boundary condition with different $D_{0}$ values.

artificial, not due to the natural decay along the downstream direction associated with the viscous dissipation. To demonstrate this point, we compute the time-averaged mean and the root-mean-square (rms) of the signal $\Psi(t)$, and denote them respectively by $\bar{\Psi}$ and $\Psi^{\prime}$. Table 4 summarizes these quantities at the outflow plane $x=10 d$ and two nearby interior planes $x=9 d$ and $9.5 d$ obtained with several $D_{0}$ values in the outflow boundary condition. At the two interior planes $x=9 d$ and $x=9.5 d$, the mean and rms of $\Psi(t)$ resulting from different $D_{0}$ values are comparable, and a slight decay in these quantities can be observed from plane $x=9 d$ to plane $x=9.5 d$. At the outflow plane, however, the mean and rms values of $\Psi(t)$ are very different with different $D_{0}$ values. The boundary condition with $D_{0}=0$ results in a dramatic decay in the mean and rms values of $\Psi(t)$ at the outflow plane. Comparison with the values in planes $x=9 d$ and $9.5 d$ suggests that this decrease at the outflow plane is too substantial to be natural. With larger $D_{0}$ values the mean and rms of $\Psi(t)$ at the outflow boundary increases, and with $D_{0}=\frac{1}{U_{0}}$ the results at the outflow plane have been significantly improved when compared with the values at the interior planes $x=9 d$ and 9.5d. When $D_{0}$ increases further to $D_{0} U_{0}=2$ and $D_{0} U_{0}=5$ the vorticity at the outflow plane becomes worse again, in the sense that the mean and rms of $\Psi(t)$ become significantly larger than those at the interior planes $x=9 d$ and $9.5 d$.

The above results suggest that the $D_{0}$ value in the open boundary condition modifies the vorticity (and the velocity derivatives in general) in a region near the outflow boundary. One should choose $\frac{1}{D_{0}}$ in accordance with the convection velocity scale of the vortices at the outflow boundary, which for the current problem is close to $U_{0}$, in order to improve the characteristics of the computed flow near the outflow boundary. A larger deviation of the chosen $\frac{1}{D_{0}}$ value from the actual vortex-convection velocity at the outflow boundary may lead to qualitatively poorer flow patterns near the outflow boundary. The value $D_{0}=0$ in general flow situations may not be the most favorable for the open boundary condition and can lead to poor flow characteristics near the outflow boundary.

The observation that a non-zero $D_{0}$ value in the current open boundary condition can result in improved 

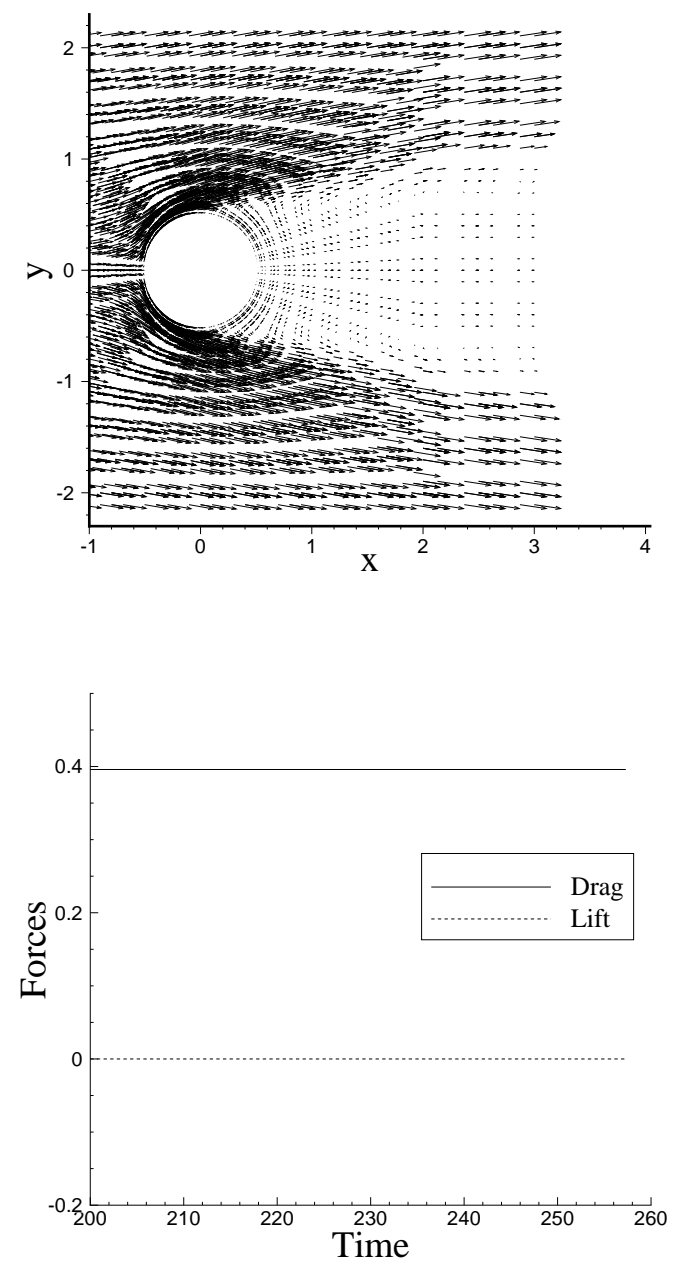

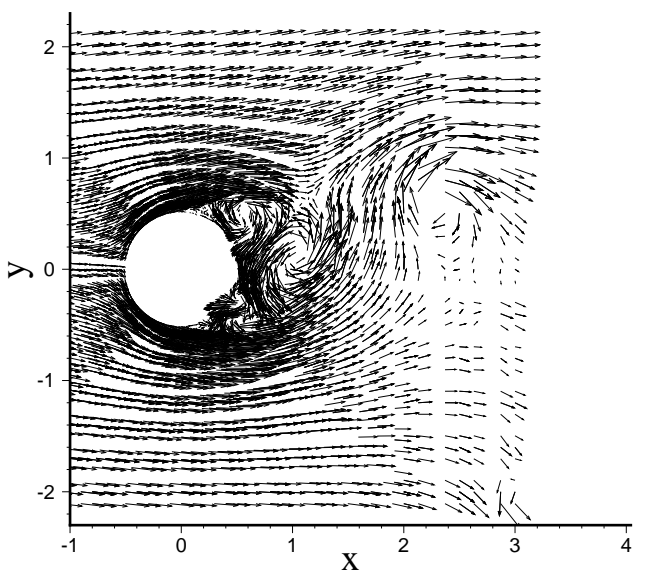

(a)

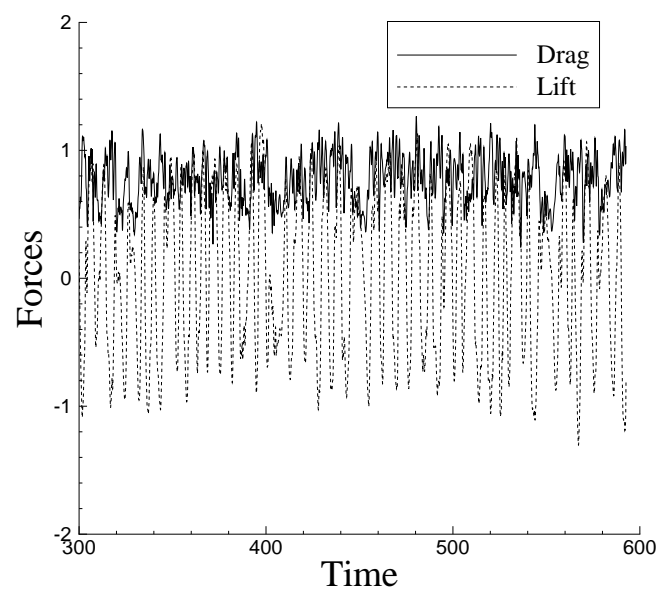

(d)

Figure 14: Cylinder flow on domain with very small wake region length $L / d=3$. (a) and (b): instantaneous velocity fields. (c) and (d): time histories of drag and lift. (a) and (c) are for $R e=500$, and (b) and (d) are for $R e=10000$. Velocity vectors are plotted on every fifth quadrature points in each direction within each element. $D_{0}=\frac{1}{U_{0}}$ in the open boundary condition.

flow patterns at the outflow boundary, when compared to $D_{0}=0$, may be understood in part based on the energy balance relation from Section 2. Let us assume that there is no external body force $(\mathbf{f}=0)$ and that $\mathbf{u}=0$ on $\partial \Omega_{d}$ for simplicity. As discussed in Section 2 , the quantity $\left(\int_{\Omega} \frac{1}{2}|\mathbf{u}|^{2}+\nu D_{0} \int_{\partial \Omega_{o}} \frac{1}{2}|\mathbf{u}|^{2}\right)$ will not grow over time (at the continuum level) when the current open boundary condition with $D_{0}>0$ is imposed on $\partial \Omega_{o}$, according to the energy balance relation (9). This provides some control over the velocity $\mathbf{u}$ on the outflow boundary $\partial \Omega_{o}$. On the other hand, if $D_{0}=0$, the energy balance only ensures that $\int_{\Omega} \frac{1}{2}|\mathbf{u}|^{2}$ does not increase over time, and the control over the velocity $\mathbf{u}$ on the outflow boundary $\partial \Omega_{o}$ is lost. Consequently, the velocity $\mathbf{u}$ can in principle take arbitrary values on $\partial \Omega_{o}$, which still comply with the energy balance. This may result in poor flow patterns at the outflow boundary. 
Finally we make some comments concerning the size of the computational domains. Thanks to the energy-stable nature of the current open boundary condition, domains with arbitrarily small downstream length can be used in computations in principle, and stable simulation results can be obtained. A smaller computational domain can be advantageous from the cost perspective, especially for situations with large Reynolds numbers. On the other hand, one should caution that an overly small computational domain can be too restrictive, so much that the main flow characteristics may be significantly modified and therefore may not be acceptable from the accuracy perspective. We use Figure 14 for illustration of these points. Here we consider a computational domain for the cylinder flow with a very small downstream length $L / d=3$, while in the other directions the domain dimensions are the same as in previous tests. Numerical experiments are performed on this domain at Reynolds numbers ranging from $R e=100$ to $R e=10000$ using the

current open boundary condition with $D_{0}=\frac{1}{U_{0}}$. Stable simulation results have been attained for all the Reynolds numbers, but the overly restrictive downstream length makes them significantly different from those obtained on larger domains. We observe that the flow field evolves into a steady state for Reynolds numbers below about $R e=1000$, while at larger Reynolds numbers it is unsteady with vortex shedding. Figure 14 shows the instantaneous velocity fields and the drag/lift histories at $R e=500$ and $R e=10000$. One can compare Figure 14(c) and Figure 4(b) to discern that the domain $L=3 d$ has completely changed the flow characteristics for $R e=500$. A comparison between Figure 14(d) and Figure 9(b) indicates that the mean drag level obtained on the domain $L=3 d$ is noticeably different from that from the larger domain for $R e=10000$. The importance of the current open boundary condition (and also those from [14, 18]) lies in that they relieve the user of the concern of backflow instability at the outflow/open boundary. Therefore, one can focus solely on other considerations such as the physical accuracy when conducting the simulations.

\subsection{Jet in Open Domain}

In this subsection we apply the current method to simulate a jet in an open domain. This is a type of flow different from that of the previous subsection. The open domain boundaries combined with the physical instability of the jet at large Reynolds numbers make this type of flows very challenging to simulate. We will again consider two-dimensional simulations.

Figure 15 is a sketch of the flow configuration. We consider a jet with an inlet diameter $d$, which is contained in a rectangular domain, $-2.5 d \leqslant x \leqslant 2.5 d$ and $0 \leqslant y \leqslant 7.5 d$. The bottom of the domain $(y=0)$ is a solid wall, and the jet inlet is located in the middle of the wall, covering $-0.5 d \leqslant x \leqslant 0.5 d$. The other three boundaries (top, left and right) of the domain are open, where the fluid can freely leave or enter the 


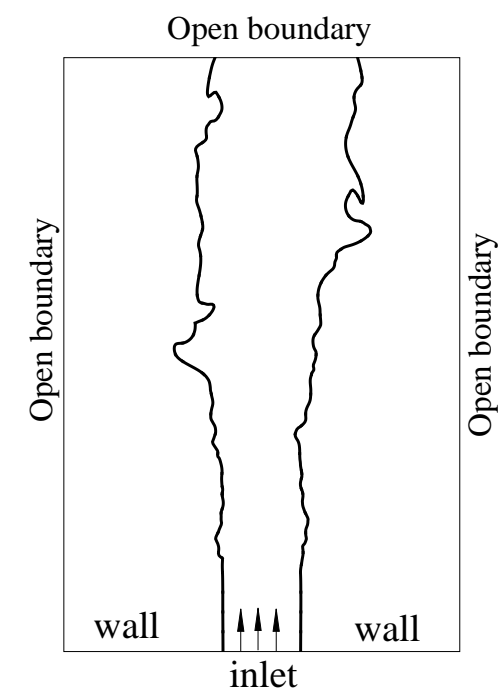

Figure 15: Configuration of jet in an open domain.

domain. We assume that the jet velocity at the inlet has the following profile

$$
\left\{\begin{array}{l}
u=0 \\
v=U_{0}\left[(H(x, 0)-H(x, d / 2)) \tanh \frac{1-\frac{2 x}{d}}{\sqrt{2} \epsilon}+(H(x,-d / 2)-H(x, 0)) \tanh \frac{1+\frac{2 x}{d}}{\sqrt{2} \epsilon}\right]
\end{array}\right.
$$

where $(u, v)$ are components of the velocity $\mathbf{u}$ in $x$ and $y$ directions, $U_{0}$ is a velocity scale, and $\epsilon=\frac{d}{40}$. $H(x, a)$ is the Heaviside step function, assuming a unit value if $x \geqslant a$ and vanishing otherwise. Note that with this profile the inlet flow has a bulk velocity $U_{0}$. We assume that there is no external body force for this problem.

We normalize all the length variables by the jet inlet diameter $d$, and all the velocity variables by $U_{0}$. The Reynolds number is therefore given by equation (44), in which $d$ and $U_{0}$ have meanings particular to this problem.

The domain has been discretized using 600 equal-sized quadrilateral spectral elements, with 20 elements in the $x$ direction and 30 elements in the $y$ direction. We impose the velocity Dirichlet condition (3) on the bottom side of the domain, with the boundary velocity $\mathbf{w}(\mathbf{x}, t)=0$ in the wall region and set according to equation (46) in the jet inlet. On the top, left and right sides of the domain we impose the open boundary condition (4) with $\mathbf{f}_{b}=0$ and $\delta=\frac{1}{100}$. The majority of simulations in this subsection are performed using $D_{0}=\frac{1}{U_{0}}$ in the open boundary condition. Several other $D_{0}$ values have also been considered in selected cases for comparison.

We integrate the Navier-Stokes equations (1a)-(1b) in time using the algorithm described in Section 2. Long-time simulations have been performed at three Reynolds numbers: $R e=2000,5000$ and 10000. In the 


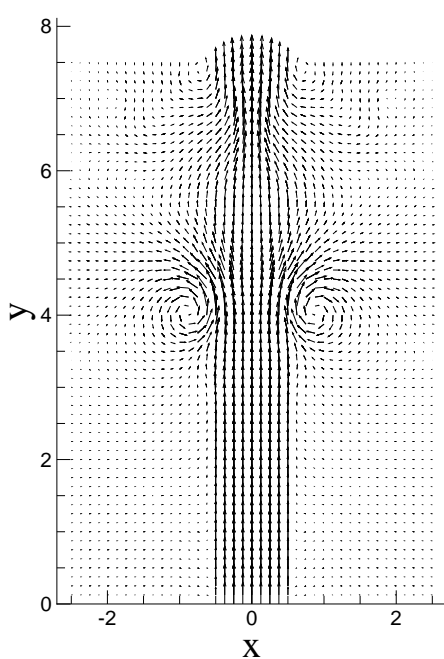

(a)
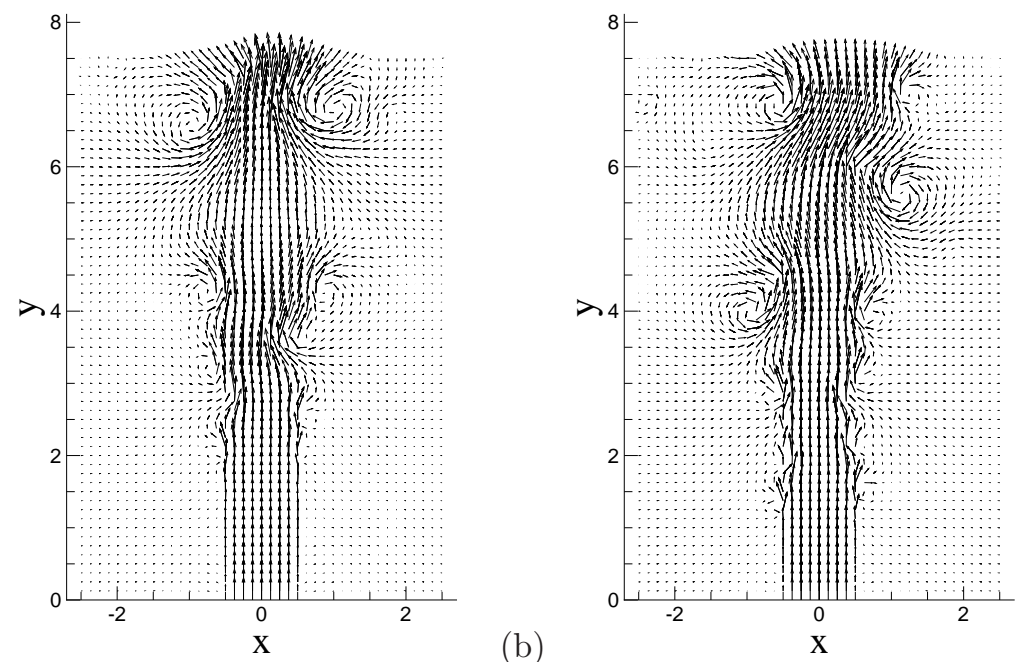

Figure 16: Jet in open domain: snapshots of instantaneous velocity fields at (a) $R e=2000,(\mathrm{~b}) R e=5000$, and (c) $R e=10000$. Velocity vectors are plotted on every ninth quadrature points in each direction within each element. Results are obtained with $D_{0}=\frac{1}{U_{0}}$ in the open boundary condition.

simulations we employ an element order 12 for each element at the two lower Reynolds numbers, and an element order 16 for each element at $R e=10000$. The non-dimensional time step size is $\frac{U_{0} \Delta t}{d}=2.5 \times 10^{-4}$ for $R e=2000$ and 5000 , and $\frac{U_{0} \Delta t}{d}=2.0 \times 10^{-4}$ for $R e=10000$.

The flow characteristics are illustrated by snapshots of the instantaneous velocity shown in Figure 16 at these Reynolds numbers. These results are obtained using $D_{0}=\frac{1}{U_{0}}$ in the open boundary condition (4). At $R e=2000$, the jet appears to be stable within a distance of at least $3 d$ downstream of the inlet (Figure 16(a)). Beyond this region, the jet becomes unstable and a pair of vortices forms, which travels downstream along with the jet and eventually crosses the upper open boundary and exits the domain. The velocity field appears symmetric with respect to the jet centerline at this Reynolds number. As the Reynolds number increases to $R e=5000$, the stable region immediately downstream of the inlet becomes shorter (approximately $2 d$, see Figure $16(\mathrm{~b})$ ), and the velocity distribution has lost the symmetry with respect to the jet centerline. Pairs of vortices can be observed to form, wrapping around the jet at different downstream locations. At $R e=10000$, The stable region downstream of the inlet becomes evern shorter (about $d$ ), and the vortices forming along the jet are notably more numerous (Figure 16(c)).

The jet simulations using the current method are long-term stable, even in the presence of backflows or vortices (see e.g. Figure 16(c)) at the open domain boundaries. This is demonstrated by Figure 17, in which we show a window of the time histories of the vertical force acting on the bottom wall for these three Reynolds numbers. Note that the horizontal force ( $x$ component) on the wall is essentially zero. These results again correspond to $D_{0}=\frac{1}{U_{0}}$ in the open boundary condition (4). One can observe that the force signals are 


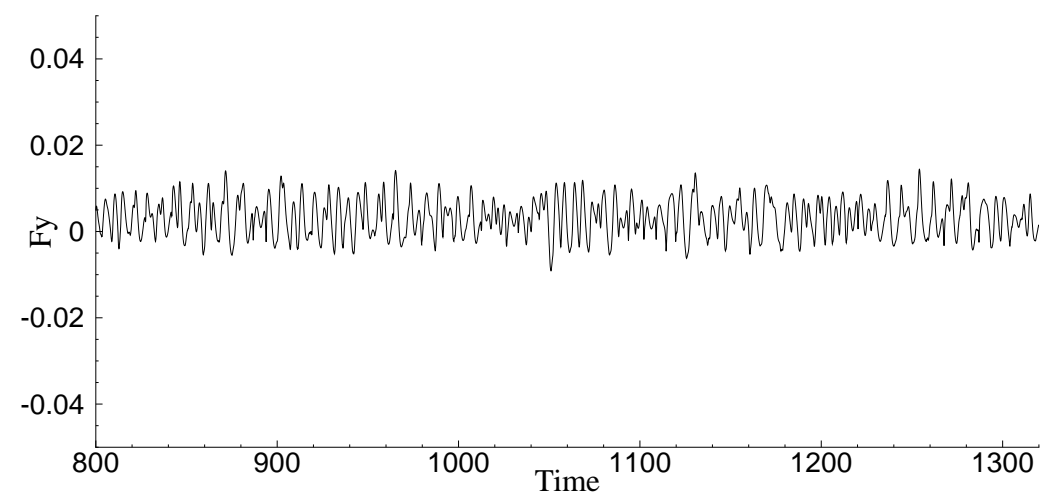

(a)

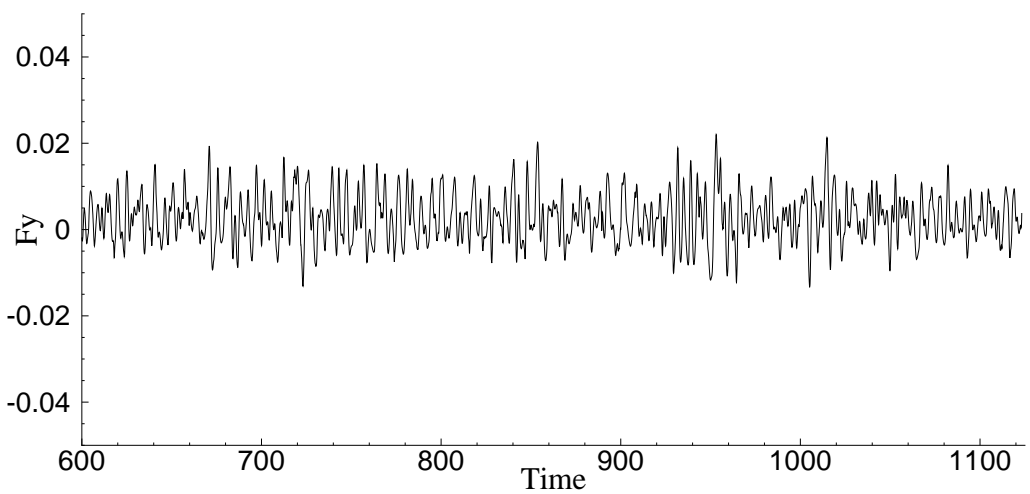

(b)

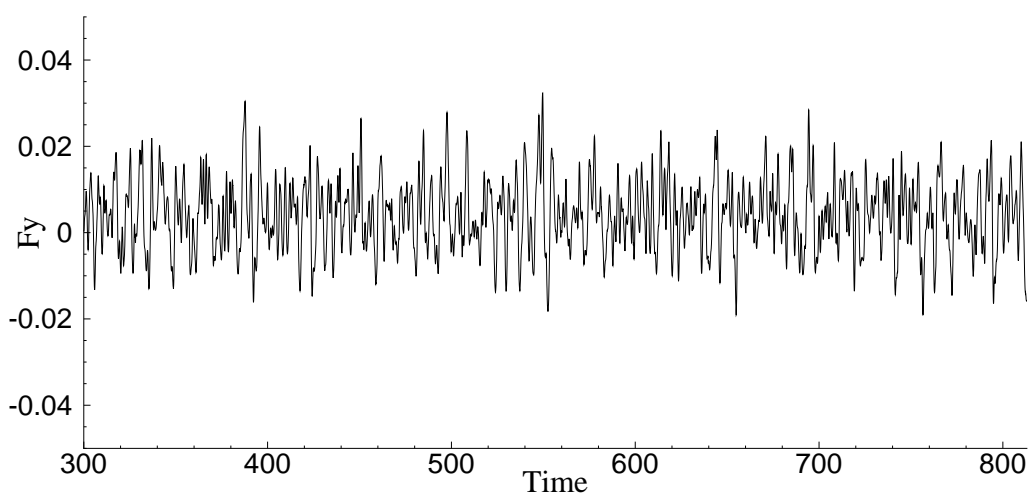

(c)

Figure 17: Jet in open domain: Time histories of the vertical force component exerting on the wall at Reynolds numbers (a) $R e=2000$, (b) $R e=5000$, and (c) $R e=10000$. Results correspond to $D_{0}=\frac{1}{U_{0}}$ in the open boundary condition. 


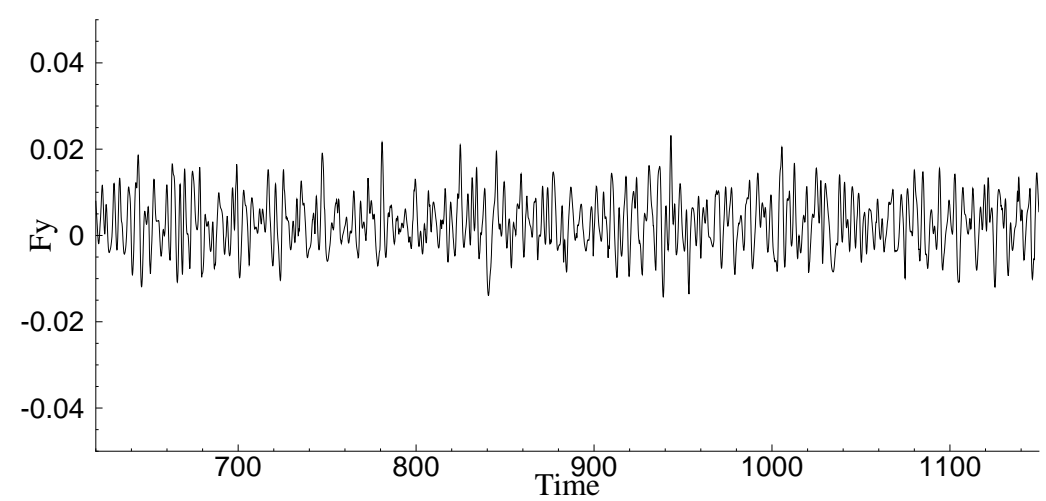

(a)

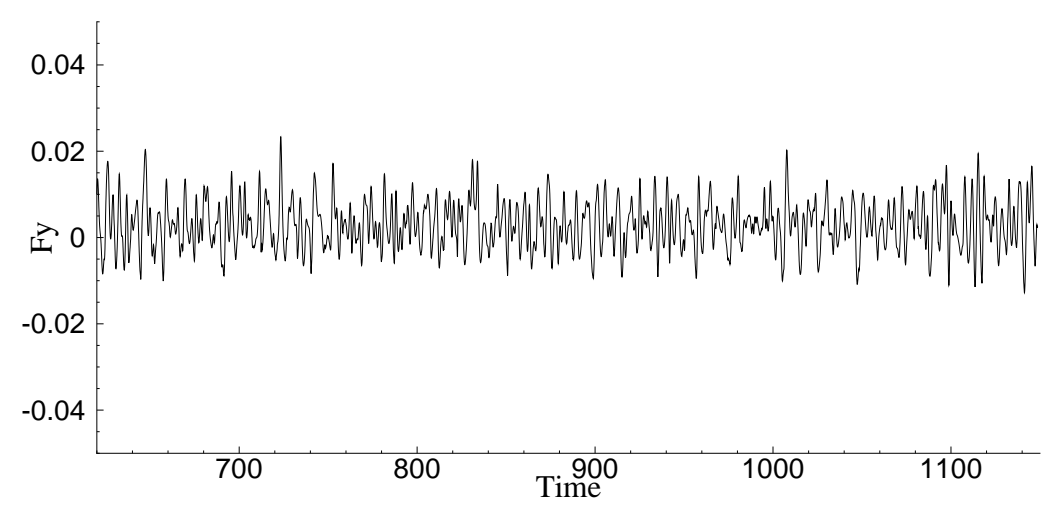

Figure 18: Jet in open domain $(R e=5000)$ : time histories of the vertical force component obtained with different $D_{0}$ values in OBC: (a) $D_{0} U_{0}=0$ and (b) $D_{0} U_{0}=2.0$. These can be compared with Figure 17(b), which corresponds to $D_{0} U_{0}=1.0$ at the same Reynolds number.

highly unsteady, and the fluctuations appear more energetic and chaotic with increasing Reynolds numbers (Figure 17(c)). The large temporal window shown here, over $500 \frac{d}{U_{0}}$ or approximately 67 flow through times, signifies the long-term stability of our simulations.

The term containing $\Theta_{0}(\mathbf{n}, \mathbf{u})$ in the open boundary condition (4) is critical to the stability of the current simulations. For comparison, we have also employed the boundary condition (10) on the open boundaries for simulations of the current problem, and observe that the computation is unstable for all the Reynolds numbers considered here. The simulations blow up instantly as the vortices reach the open boundaries.

The results shown so far have been obtained using $D_{0}=\frac{1}{U_{0}}$ in the open boundary condition (4). We next concentrate on the Reynolds number $R e=5000$, and look into the effect of $D_{0}$ on the simulation results. We have considered several $D_{0}$ values in the open boundary condition: $D_{0} U_{0}=0,0.5,1.0$, and 2.0. Figure 18 shows time histories of the vertical force on the wall at $R e=5000$ obtained using $D_{0}=0$ and $D_{0}=\frac{1}{U_{0} / 2}$ in the open boundary condition (4). These plots can be compared with Figure 17(b), which corresponds to $D_{0}=\frac{1}{U_{0}}$ at the same Reynolds number. These force signals appear qualitatively similar. 


\begin{tabular}{lccc}
\hline$D_{0} U_{0}$ & $\bar{f}_{x}$ & $\bar{f}_{y}$ & $f_{y}^{\prime}$ \\
0.0 & $-1.621 E-6$ & $2.891 E-3$ & $6.065 E-3$ \\
0.5 & $1.203 E-6$ & $2.864 E-3$ & $5.713 E-3$ \\
1.0 & $-9.599 E-7$ & $2.729 E-3$ & $5.549 E-3$ \\
2.0 & $2.829 E-7$ & $2.910 E-3$ & $5.541 E-3$ \\
\hline
\end{tabular}

Table 5: Mean and rms forces on the wall for the jet problem at $R e=5000$ obtained using different $D_{0}$ values in OBC. $\bar{f}_{x}$ and $\bar{f}_{y}$ denote the time-averaged mean forces in horizontal and vertical directions, respectively. $f_{y}^{\prime}$ is the rms force in the vertical direction.

To provide a quantitative comparison, we have listed in Table 5 the time-averaged mean and rms forces acting on the wall corresponding to different $D_{0}$ values in the open boundary condition. The mean force in the horizontal direction $\left(\bar{f}_{x}\right)$ is essentially zero for all cases. Both the mean and rms forces in the vertical direction obtained using different $D_{0}$ values are close, with a maximum difference of about $6.6 \%$ for the mean vertical force and a maximum difference of about $9.5 \%$ for the rms vertical force. The $D_{0}$ effect on the global flow quantities (such as forces) observed here for the jet flow is approximately in line with the observations for the cylinder flow in Section 3.2. But the differences observed for this problem appear slightly larger.

\section{Concluding Remarks}

The main contributions of the current paper are two-fold:

- We have presented a new type of energy-stable open boundary condition for incompressible flow simulations. This boundary condition ensures the energy stability of the system, and in some sense can be analogized to the usual convective boundary condition. The current open boundary condition can be reduced to that of [18] if the inertia term involved herein vanishes (by setting $D_{0}=0$ ). Note that the current boundary condition can be generalized to a family of convective-like energy-stable open boundary conditions with an idea similar to that employed in [18]; see the remarks in Section 2.1.

- We have presented an efficient numerical algorithm for the proposed open boundary condition. The key issue here lies in the numerical treatment of the inertia term involved in the current open boundary condition. Our algorithm combines a rotational velocity-correction type splitting strategy for the Navier-Stokes equations with two different implicit approximations of the inertia term in the open boundary condition for the pressure and velocity sub-steps. The algorithm leads to Robin-type conditions for both the discrete pressure and the discrete velocity on the outflow/open boundary.

We would like to point out that the open boundary condition proposed herein can be considered as the energy-stable version of a combined traction-free and convective boundary condition (see equation (10)). As discussed in Section 2.1, this condition will be reduced to the traction-free condition if the inertia term 
vanishes (i.e. $D_{0}=0$ ), and if $p=0$ is imposed on the outflow boundary it will be reduced essentially to the usual convective boundary condition.

The method developed in the current work for dealing with outflow/open boundaries exhibits favorable properties when compared with those of $[14,18]$ in at least two aspects. First, it can lead to qualitatively smoother flow patterns at or near the outflow boundary, because the current boundary condition (when $\left.D_{0}>0\right)$ provides a control over the velocity on the outflow boundary. This has been demonstrated by the numerical simulations of Section 3. Second, it provides a simpler implementation (with $D_{0}>0$ ) with $C^{0}$ spectral-element and finite-element type methods. This is because the current method essentially imposes a discrete Robin-type condition for both the velocity and the pressure on the outflow boundary, and requires only an update to the coefficient matrix by a surface integral in the implementation. In contrast, with the methods of $[14,18]$ a pressure Dirichlet type condition is imposed on the outflow boundary, and a projection to the $H^{1}\left(\partial \Omega_{o}\right)$ space of the pressure Dirichlet data will be required with $C^{0}$ elements in the implementation. This projection is more involved than the evaluation of the surface integral required by the current method.

On the other hand, certain aspects of the method can and remain to be improved. The convective-like energy-stable open boundary condition developed here is more complicated in form, due to the inertia term, compared to the boundary conditions from $[14,18]$. The Robin-type conditions for the discrete pressure and velocity on the outflow boundary, which arise from the implicit treatment of this inertia term, may complicate the implementation and present an issue for certain types of spatial discretization methods.

The effectiveness of the current method has been demonstrated by extensive numerical simulations. Comparison with the experimental data shows the accuracy of the current method. At higher Reynolds numbers when backflows and strong vortices occur at the outflow/open boundaries, numerical results have demonstrated the long-term stability using the current method. It is observed that the method allows the vortices to discharge from the domain in a fairly natural fashion, even at quite high Reynolds numbers (up to $R e=10000$ tested here). We would like to point out that the flow tests presented here are in two dimensions. The method remains to be tested with three-dimensional turbulent flows.

The several forms of convective-like energy-stable open boundary conditions mentioned in Section 2.1 have a correspondence to those boundary conditions developed in [18]. If $D_{0}=0$ these several forms will reduce to those of [18]. In [18] the different forms of boundary conditions have been studied closely, and it is observed that the form "OBC-C" appears to be the most favorable among them. Note that the convectivelike energy-stable open boundary condition proposed here, equation (4), reduces to the condition OBC-C of [18] if $D_{0}=0$. In light of the study and observations of [18], we expect that the boundary condition given by (4) will be more favorable than the other forms of convective-like energy-stable open boundary conditions from Section 2.1. 
We anticipate that the presented method will be instrumental in numerical studies of wakes, jets, shear layers, and other types of flows involving physically unbounded domains, especially for high Reynolds numbers. It would be very interesting to extend the idea and develop analogous boundary conditions for moving domains such as in an arbitrary Lagrangian Eulerian context. This would be important and useful to applications such as vortex/flow induced vibrations. Future research will address such and related problems.

\section{Acknowledgement}

This work is partially supported by NSF (DMS-1318820) and ONR (N000141110028). The author would like to thank the anonymous referees for valuable suggestions and comments.

\section{References}

[1] Y. Bazilevs, J.R. Hohean, T.J.R. Hughes, R.D. Moser, and Y. Zhang. Patient-specific isogeometric fluid-structure interaction analysis of theracic aortic blood flow due to impantation of the jarvik 2000 left ventricular assist device. Comput. Methods Appl. Mech. Engrg., 198:3534-3550, 2009.

[2] P. Beaudan and P. Moin. Numerical experiments on the flow past a circular cylinder at sub-critical reynolds numbers, 1994. Report No. TF-62, Thermal Sciences Division, Department of Mechanical Engineering, Stanford University.

[3] M. Behr, J. Liou, R. Shih, and T.E. Tezduyar. Vorticity-streamfunction formulation of unsteady incompressible flow past a cylinderl sentivity of the computed flow field to the location of the outflow boundary. International Journal for Numerical Methods in Fluids, 12:323-342, 1991.

[4] C. Bertoglio and A. Caiazzo. A tangential regularization method for backflow stabilization in hemodynamics. Journal of Computational Physics, 261:162-171, 2014.

[5] M. Braza, P. Chassaing, and H. Ha Minh. Numerical study and physical analysis of the pressure and velocity fields in the near wake of a circular cylinder. Journal of Fluid Mechanics, 165:79-130, 1986.

[6] C.-H. Bruneau and P. Fabrie. Effective downstream boundary conditions for incompressible NavierStokes equations. International Journal for Numerical Methods in Fluids, 19:693-705, 1994.

[7] C.-H. Bruneau and P. Fabrie. New efficient boundary conditions for incompressible navier-stokes equations: a well-posedness result. Mathematical Modeling and Numerical Analysis, 30:815-840, 1996.

[8] J. Craske and M. van Reeuwijk. Robust and accurate open boundary conditions for incompressible turbulent jets and plumes. Computers $\& 3$ Fluids, 86:284-297, 2013. 
[9] N.K. Delany and N.E. Sorenson, 1953. NACA Tech. Note 3038.

[10] S. Dong. On imposing dynamic contact-angle boundary conditions for wall-bounded liquid-gas flows. Computer Methods in Applied Mechanics and Engineering, 247:179-200, 2012.

[11] S. Dong. An efficient algorithm for incompressible N-phase flows. Journal of Computational Physics, 276:691-728, 2014.

[12] S. Dong. An outflow boundary condition and algorithm for incompressible two-phase flows with phase field approach. Journal of Computational Physics, 266:47-73, 2014.

[13] S. Dong and G.E. Karniadakis. DNS of flow past stationary and oscillating cylinder at $R e=10,000$. J. Fluids Struct., 20:14-23, 2005.

[14] S. Dong, G.E. Karniadakis, and C. Chryssostomidis. A robust and accurate outflow boundary condition for incompressible flow simulations on severely-truncated unbounded domains. Journal of Computational Physics, 261:83-105, 2014.

[15] S. Dong, G.E. Karniadakis, A. Ekmekci, and D. Rockwell. A combined DNS-PIV study of the turbulent near wake. J. Fluid Mech., 569:185-207, 2006.

[16] S. Dong and J. Shen. An unconditionally stable rotational velocity-correction scheme for incompressible flows. J. Comput. Phys., 229:7013-7029, 2010.

[17] S. Dong and J. Shen. A time-stepping scheme involving constant coefficient matrices for phase field simulations of two-phase incompressible flows with large density ratios. Journal of Computational Physics, 231:5788-5804, 2012.

[18] S. Dong and J. Shen. A pressure-correction scheme for generalized form of energy-stable open boundary conditions for incompressible flows. Journal of Computational Physics, 291:254-278, 2015.

[19] M.S. Engelman and M.-A. Jamnia. Transient flow past a circular cylinder: A benchmark solution. International Journal for Numerical Methods in Fluids, 11:985-1000, 1990.

[20] M. Feistauer and T. Neustupa. On the existence of a weak solution of viscous incompressible flow past a cascade of profiles with an arbitrarily large inflow. J. Math. Fluid Mech., 15:701-715, 2013.

[21] R.K. Finn. Determination of the drag on a cylinder at low Reynolds numbers. Journal of Applied Physics, 24:771-773, 1953. 
[22] M.Y. Forestier, R. Pasquetti, R. Peuret, and C. Sabbah. Spatial development of wakes using a spectral multi-domain method. Applied Numerical Mathematics, 33:207-216, 2000.

[23] L. Formaggia, J.-F. Gerbeau, F. Nobile, and A. Quarteroni. Numerical treatment of defective boundary conditions for the navier-stokes equations. SIAM J. Numer. Anal., 40:376-401, 2002.

[24] R. Franke, W. Rodi, and B. Schonung. Numerical calculation of laminar vortex-shedding flow cylinders. Journal of Wind Engineering and Inductrial Aerodynamics, 35:237-257, 1990.

[25] D.K. Gartling. A test problem for outflow boundary conditions - flow over a backward-facing step. International Journal for Numerical Methods in Fluids, 11:953-967, 1990.

[26] N.S. Ghaisas, D.A. Shetty, and S.H. Frankel. Large eddy simulation of turbulent horizontal buoyant jets. Journal of Turbulence, 16:772-808, 2015.

[27] V. Gravemeier, A. Comerford, L. Yoshihara, M. Ismail, and W.A. Wall. A novel formulation for Neumann inflow boundary conditions in biomechanics. International Journal for Numeical Methods in Biomedical Engineering, 28:560-573, 2012.

[28] P.M. Gresho. Incompressible fluid dynamics: some fundamental formulation issues. Annual Review of Fluid Mechanics, 23:413-453, 1991.

[29] L. Grinberg and G.E. Karniadakis. Outflow boundary conditions for arterial networks with multiple outlets. Annals of Biomedical Engineering, 36:1496-1514, 2008.

[30] J.L. Guermond, P. Minev, and J. Shen. Error analysis of pressure-correction schemes for the timedependent stokes equations with open boundary conditions. SIAM J. Numer. Anal., 43:239-258, 2005.

[31] J.L. Guermond and J. Shen. A new class of truly consistent splitting schemes for incompressible flows. J. Comput. Phys., 192:262-276, 2003.

[32] J.L. Guermond and J. Shen. Velocity-correction projection methods for incompressible flows. SIAM J. Numer. Anal., 41:112-134, 2003.

[33] N. Hasan, S.F. Anwer, and S. Sanghi. On the outflow boundary condition for external incompressible flows: a new approach. Journal of Computational Physics, 206:661-683, 2005.

[34] J.G. Heywood, R. Rannacher, and S. Turek. Artificial boundaries and flux and pressure: conditions for the incompressible navier-stokes equations. International Journal for Numerical Methods in Fluids, 22:325-352, 1996. 
[35] R.R. Hwang and S.H. Lin. On laminar wakes behind a circular cylinder in stratified fluids. ASME Journal of Fluids Engineering, 114:20-28, 1992.

[36] M. Ismail, V. Gravemeier, A. Comerford, and W.A. Wall. A stable approach for coupling multidimensional cardiovascular and pulmonary networks based on a novel pressure-flow rate or pressure-only neumann boundary condition formulation. International Journal for Numerical Methods in Biomedical Engineering, 30:447-469, 2014.

[37] G. Jin and M. Braza. A nonreflecting outlet boundary condition for incompressible unsteady navierstokes calculations. Journal of Computational Physics, 107:239-253, 1993.

[38] B.C.V. Johansson. Boundary conditions for open boundaries for the incompressible navier-stokes equation. Journal of Computational Physics, 105:233-251, 1993.

[39] G.E. Karniadakis. Numerical simulation of forced convection heat transfer from a cylinder in crossflow. International Journal of Heat and Mass Transfer, 31:107-118, 1988.

[40] G.E. Karniadakis, M. Israeli, and S.A. Orszag. High-order splitting methods for the incompressible Navier-Stokes equations. J. Comput. Phys., 97:414-443, 1991.

[41] G.E. Karniadakis and S.J. Sherwin. Spectral/hp element methods for computational fluid dynamics, 2nd edn. Oxford University Press, 2005.

[42] J. Keskar and D.A. Lyn. Computations of a laminar backward-facing step flow at re $=800$ with a spectral domain decomposition method. International Journal for Numerical Methods in Fluids, 29:411-427, 1999.

[43] A.G. Kravchenko, P. Moin, and K. Shariff. B-spline method and zonal grids for simulations of complex turbulent flows. Journal of Computational Physics, 151:757-789, 1999.

[44] M. Lanzendorfer and J. Stebel. On perssure boundary conditions for steady flows of incompressible fluids with pressure and shear rate dependent viscosities. Applications of Mathematics, 56:265-285, 2011.

[45] J.M. Leone. Open boundary condition symposium benchmark solution: stratified flow over a backwardfacing step. International Journal for Numerical Methods in Fluids, 11:969-984, 1990.

[46] J. Liu. Open and traction boundary conditions for the incompressible navier-stokes equations. Journal of Computational Physics, 228:7250-7267, 2009. 
[47] J.-G. Liu, J. Liu, and R.L. Pego. Stability and convergence of efficient Navier-Stokes solvers via a commutator estimate. Comm. Pure Appl. Math., LX:1443-1487, 2007.

[48] X. Ma, G.-S. Karamanos, and G.E. Karniadakis. Dynamics and low-dimensionality of a turbulent near wake. Journal of Fluid Mechanics, 410:29-65, 2000.

[49] J.R. Meneghini and P.W. Bearman. Numerical simulation of high amplitude oscillatory-flow about a circular cylinder using a discrete vortex method. In AIAA Shear Flow Conference, Orlando, FL. AIAA Paper 93-3288, pages 1-11, 1993.

[50] M.E. Moghadam, Y. Bazilevs, T.-Y. Hsia, I.E. Vignon-Clementel, and A.L. Marsden. A comparison of outlet boundary treatments for prevention of backflow divergence with relevance to blood flow simulations. Comput. Mech., 48:277-291, 2011.

[51] D.J. Newman and G.E. Karniadakis. Direct numerical simulations of flow over a flexible cable. In Proceedings of 6th International Conference on Flow-Induced Vibrations, pages 193-203, 1995.

[52] D.J. Newman and G.E. Karniadakis. Simulations of flow over a flexible cable: A comparison of forced and flow-induced vibration. Journal of Fluids and Structures, 10:439-454, 1996.

[53] C. Norberg. Fluctuating lift on a circular cylinder: review and new measurements. Journal of Fluids and Structures, 17:57-96, 2003.

[54] J. Nordstrom, K. Mattsson, and C. Swanson. Boundary conditions for a divergence free velocity-free formulation of the navier-stokes equations. Journal of Computational Physics, pages 874-890, 2007.

[55] M.A. Olshanskii and V.M. Staroverov. On simulation of outflow boundary conditions in finite difference calculations for incompressible fluid. International Journal for Numerical Methods in Fluids, 33:499-534, 2000.

[56] I. Orlanski. A simple boundary condition for unbounded hyperbolic flows. Journal of Computational Physics, 21:251-269, 1976.

[57] T.C. Papanastasiou, N. Malamataris, and K. Ellwood. A new outflow boundary condition. International Journal for Numerical Methods in Fluids, 14:587-608, 1992.

[58] H. Persillon and M. Braza. Physical analysis of the transition to turbulence in the wake of a circular cylinder by three-dimensional navier-stokes simulation. Journal of Fluid Mechanics, 365:23-88, 1998. 
[59] A. Porpora, P. Zunino, C. Vergara, and M. Piccinelli. Numerical treatment of boundary conditions to replace branches in hemodynamics. International Journal of Numerical Methods in Biomedical Engineering, 28:1165-1183, 2012.

[60] A. Poux, S. Glockner, and M. Azaiez. Improvements on open and traction boundary conditions for navier-stokes time-splitting methods. Journal of Computational Physics, 230:4011-4027, 2011.

[61] A. Roshko. Experiments on the flow past a circular cylinder at very high Reynolds numbers. Journal of Fluid Mechanics, 10:345-356, 1961.

[62] M.R. Ruith, P. Chen, and E. Meiburg. Development of boundary conditions for direct numerical simulations of three-dimensional vortex breakdown phenomena in semi-infinite domains. Computers $\mathscr{G}$ Fluids, 33:1225-1250, 2004.

[63] R.L. Sani and P.M. Gresho. Resume and remarks on the open boundary conidtion minisymposium. International Journal for Numerical Methods in Fluids, 18:983-1008, 1994.

[64] S.J. Sherwin and G.E. Karniadakis. A triangular spectral element method: applications to the incompressible navier-stokes equations. Comput. Meth. Appl. Mech. Engrg., 123:189-229, 1995.

[65] A. Sommerfeld. Partial Differential Equations in Physics. Academic Press, 1949.

[66] S. Tang and N. Audry. On the symmetry breaking instability leading to vortex shedding. Physics of Fluids, 9:2550-2561, 1997.

[67] S. Taylor, J. Rance, and J.O. Medwell. A note on the imposition of traction boundary conditions when using the FEM for solving incompressible flow problems. Communications in Applied Numerical Methods, 1:113-121, 1985.

[68] L.J.P. Timmermans, P.D. Minev, and F.N. van de Vosse. An approximate projection scheme for incompressible flow using spectral elements. Int. J. Numer. Meth. Fluids, 22:673-688, 1996.

[69] D.J. Tritton. Experiments on the flow past a circular cylinder at low Reynolds numbers. Journal of Fluid Mechanics, 6:547, 1959.

[70] S.S. Varghese, S.H. Frankel, and P.F. Fischer. Direct numerical simulation of stenotic flows. Part 1. steday flow. Journal of Fluid Mechanics, 582:253-280, 2007.

[71] C. Wieselsberger. Recent statements on the laws of liquid and air resistancy. Physikalische Zeitschrift, 22:321-328, 1921. 
[72] C.H.K. Williamson. Vortex dynamics in a cylinder wake. Annual Review of Fluid Dynamics, 28:477-539, 1996.

[73] H.Q. Zhang, U. Fey, B.R. Noack, M. Konig, and H. Engelmann. On the transition of the cylinder wake. Physics of Fluids, 7:779-793, 1995.

[74] J. Zhang and C. Dalton. A three-dimensional simulation of a steady approach flow past a circular cylinder at low reynolds number. International Journal of Numerical Methods in Fluids, 26:1003-1022, 1998.

[75] X. Zheng and S. Dong. An eigen-based high-order expansion basis for structured spectral elements. Journal of Computational Physics, 230:8573-8602, 2011. 